\title{
Periodic Solutions of Certain Differential Equations with Piecewise Constant Argument
}

\author{
James Guyker \\ Department of Mathematics, SUNY College at Buffalo, 1300 Elmwood Avenue, Buffalo, NY 14222-1095, USA \\ Correspondence should be addressed to James Guyker; guykerj@buffalostate.edu
}

Received 30 March 2015; Accepted 27 May 2015

Academic Editor: Harvinder S. Sidhu

Copyright (C) 2015 James Guyker. This is an open access article distributed under the Creative Commons Attribution License, which permits unrestricted use, distribution, and reproduction in any medium, provided the original work is properly cited.

Existence criteria are derived for the eventually periodic solutions of a class of differential equations with piecewise constant argument whose solutions at consecutive integers satisfy nonlinear recurrence relations. The proof characterizes the initial values of periodic solutions in terms of the coefficients of the resulting difference equations. Sufficient conditions for the unboundedness, boundedness, and symmetry of general solutions also follow from the corresponding properties of the difference equations.

\section{Introduction}

Since the seminal works of Shah and Wiener [1] and Cooke and Wiener [2], differential equations with piecewise constant arguments of the form

$$
x^{\prime}(t)=f(t, x(t), x([t])),
$$

where $f$ is continuous and $[\cdot]$ is the greatest integer function, have been treated widely in the literature and applied to certain biomedical models (see [3-7] and references therein). Continuity of the solutions of these equations implies recurrence relations for the values of solutions at consecutive integers. Therefore, there is a natural interplay between properties of these differential equations and properties of difference equations.

In this paper, we consider a class of equations of the above form but where $f$ is discontinuous: the chaotic and eventually periodic behavior and symmetry of solutions of initial-value problems of the form

$$
x^{\prime}(t)=A x(t)+B x([t])+C F(x([t])), \quad x(0)=x_{0}
$$

on $[0, \infty)$ are determined, where $A, B$, and $C$ are constants; $F: \mathbb{R} \rightarrow \mathbb{R}$ is defined by

$$
F(x) \equiv \begin{cases}0, & \text { if } x \leq \lambda \\ 1, & \text { if } x>\lambda\end{cases}
$$

for some positive number $\lambda$, and the sequence $x(n)(n=$ $0,1, \ldots)$ satisfies a nonlinear difference equation. As in the case when $f$ is continuous, by a solution of (2), we mean a function $x(t)$ that is defined on $[0, \infty)$ with these properties:

(1) $x(t)$ is continuous on $[0, \infty)$

(2) The derivative $x^{\prime}(t)$ exists at each point $t$ in $[0, \infty)$, with the possible exception of the points $[t]$ in $[0, \infty)$, where one-sided derivatives exist.

(3) $x(t)$ satisfies (2) on $[n, n+1)$ for each nonnegative integer $n$.

Specifically, since

$$
\frac{d}{d t}\left(x(t) e^{-A t}\right)=B x([t]) e^{-A t}+C F(x([t])) e^{-A t},
$$

it follows that if $n$ is a positive integer and $n-1 \leq t^{\prime}<n$, then

$$
\begin{aligned}
& x\left(t^{\prime}\right) e^{-A t^{\prime}}-x(n-1) e^{-A(n-1)} \\
& \quad=(B x(n-1)+C F(x(n-1))) \int_{n-1}^{t^{\prime}} e^{-A t} d t .
\end{aligned}
$$

Letting $t^{\prime}$ increase to $n$, we have the difference equation

$$
x(n)=a^{*} x(n-1)-b^{*} F(x(n-1)),
$$


where $x(0)=x_{0}$,

$$
\begin{aligned}
& a^{*} \equiv \begin{cases}e^{A}+B \frac{e^{A}-1}{A}, & \text { if } A \neq 0 \\
1+B, & \text { if } A=0,\end{cases} \\
& b^{*} \equiv \begin{cases}-C \frac{e^{A}-1}{A}, & \text { if } A \neq 0 \\
-C, & \text { if } A=0 .\end{cases}
\end{aligned}
$$

Therefore, the unique solution to (2) is

$$
\begin{aligned}
x(t)= & \left(e^{A(t-[t])}+B \int_{[t]}^{t} e^{A(t-s)} d s\right) x([t]) \\
& +C\left(\int_{[t]}^{t} e^{A(t-s)} d s\right) F(x([t])),
\end{aligned}
$$

where

$$
\int_{[t]}^{t} e^{A(t-s)} d s= \begin{cases}\frac{e^{A(t-[t])}-1}{A}, & \text { if } A \neq 0 \\ t-[t], & \text { if } A=0\end{cases}
$$

Moreover, $x(t)$ satisfies (1)-(3) above since $x(t)$ is continuous on $[0, \infty)$ with left derivatives

$$
x^{\prime}\left(n^{-}\right)=A x(n)+B x(n-1)+C F(x(n-1))
$$

at integers $n>0$, with right derivatives

$$
x^{\prime}\left(n^{+}\right)=(A+B) x(n)+C F(x(n))
$$

at integers $n \geq 0$, and more generally with derivatives at nonintegral $t$ given by

$$
x^{\prime}(t)=((A+B) x([t])+C F(x([t]))) e^{A(t-[t])} .
$$

We recall that the solution $x=x(t)$ to (2) is oscillatory if it has arbitrarily large zeros [3]. Accordingly, a sequence $x_{n}(n=$ $0,1, \ldots)$ is oscillatory if there exists a subsequence $x_{n_{k}}$ such that $x_{n_{k}} x_{n_{k+1}} \leq 0$ for all $k$. Moreover, a stationary state of $x_{n}$ is a term $x_{i}$ such that $x_{i}=x_{i+1}$ [8]. The following holds for solutions of (2).

Proposition 1. Let $x=x(t)$ be given by (8), where $x(n)(n=$ $0,1, \ldots)$ satisfies $(6)$.

(1) If $a^{*} \neq 1$ and $x(i)$ is a stationary state of $x(n)$, then either $x=x(t)=0$ for all $t \geq i$ or $x=x(t)=b^{*} /\left(a^{*}-\right.$ 1) for all $t \geq i$.

(2) Let $a^{*}>1$.

(a) If $x=x(t)$ is oscillatory, then $x(n)$ is oscillatory with stationary state 0 .

(b) If $x(n)$ diverges to $\infty$, then $\lim _{t \rightarrow \infty} x(t)=\infty$.

(c) If $x(n)$ diverges to $-\infty$, then $\lim _{t \rightarrow \infty} x(t)=-\infty$.
Proof. (1) Let $a^{*} \neq 1$ and let $x(i)=x(i+1)$. Suppose first that $x(i) \leq \lambda$. Then $x(i+1)=a^{*} x(i)=x(i)$ so $x(i)=x(i+1)=0$ since $a^{*} \neq 1$. By induction, $x(i+j)=0<\lambda$ and $F(x(i+j))=0$ for all integers $j \geq 0$; and, by (8), $x(t)=0$ for all $t \geq i$.

On the other hand, assume $x(i)>\lambda$. Then $x(i+1)=$ $a^{*} x(i)-b^{*}=x(i)$ so $x(i)=x(i+1)=b^{*} /\left(a^{*}-1\right)>\lambda$ since $a^{*} \neq 1$. Thus $x(i+2)=a^{*} x(i+1)-b^{*}=b^{*} /\left(a^{*}-1\right)>\lambda$ and, by induction, $x(i+j)=b^{*} /\left(a^{*}-1\right)$ for all integers $j \geq 0$. Since $b^{*} /\left(a^{*}-1\right)=-C /(A+B)$ and $F\left(b^{*} /\left(a^{*}-1\right)\right)=1$, it follows from (8) that $x(t)=b^{*} /\left(a^{*}-1\right)$ for all $t \geq i$.

(2) Let $a^{*}>1$. Then $B>-A$ and in (8) we have

$$
e^{A(t-[t])}+B \int_{[t]}^{t} e^{A(t-s)} d s>1
$$

(a) Suppose by way of contradiction that $x=x(t)$ is oscillatory but 0 is not a stationary state of $x(n)$. It follows that $x(n-1) \neq 0$ for all positive integers $n$.

Assume first that $x(n-1)<0$ for some integer $n \geq 1$. Then $F(x(n-1))=0$ and $x(n)=a^{*} x(n-1)<0<\lambda$. By induction, $x([t])<0$ and $F(x([t]))=0$ for all $t \geq n-1$.

Let $n-1 \leq[t]<t<[t]+1$. By (8) and (13), $x(t)<0$ for all $t \geq n-1$; and thus $x=x(t)$ is not oscillatory in this case, a contradiction.

Therefore, assume that $x(n-1)>0$ for all positive integers $n$. Let $n$ be a positive integer. We show that $x(t)>0$ for all $t$ in the interval $(n-1, n)$ : note that $x^{\prime}(t)=x^{\prime}\left(n-1^{+}\right) e^{A(t-[t])}$.

Suppose that $x^{\prime}\left(n-1^{+}\right)=0$. Then $x^{\prime}(t)=0$ and

(i) if $A \neq 0$, then, by (2),

$$
x(t)=-\frac{B x(n-1)+C F(x(n-1))}{A}=x(n-1)>0
$$

for all $t$ in $(n-1, n)$;

(ii) if $A=0$, then, by (2), $B x(n-1)+C F(x(n-1))=0$ and, by (8),

$$
\begin{aligned}
x(t)= & (1+B(t-[t])) x(n-1) \\
& +C(t-[t]) F(x(n-1))=x(n-1)>0
\end{aligned}
$$

for all $t$ in $(n-1, n)$.

If $x^{\prime}\left(n-1^{+}\right)>0$, then $x(t)$ is strictly increasing on $(n-1, n)$ and $x(n-1)>0$, so $x(t)>0$ for all $t$ in $(n-1, n)$ by continuity.

If $x^{\prime}\left(n-1^{+}\right)<0$, then $x(t)$ is strictly decreasing on $(n-$ $1, n)$, but $x(t)$ is still positive on $(n-1, n)$ by continuity since $x(n-1)$ and $x(n)$ are both positive.

Therefore, $x(t)>0$ for all $t$ in $[n-1, n]$ (and hence for all $t$ ). Thus $x=x(t)$ is not oscillatory in this case, contrary to our assumption.

(b) Suppose that $\lim _{n \rightarrow \infty} x(n)=\infty$. There exists $N$ such that $x(n)>\lambda$ for all integers $n \geq N$. Let $[t] \geq N$. Then $F(x([t]))=1$ and, by (8) and (13),

$$
x(t) \geq x([t])+C \int_{[t]}^{t} e^{A(t-s)} d s .
$$


Thus, if $C \geq 0$, then $x(t) \geq x([t])$. And if $C<0$, then $x(t) \geq x([t])-b^{*}$ since

$$
0 \leq \frac{e^{A(t-[t])}-1}{A} \leq \frac{e^{A}-1}{A}
$$

when $A \neq 0$ and $t-[t]<1$ otherwise. Therefore, $\lim _{t \rightarrow \infty} x(t)=\infty$.

(c) Suppose that $\lim _{n \rightarrow \infty} x(n)=-\infty$. There exists $N$ such that $x(n) \leq 0$ for all integers $n \geq N$. Let $[t] \geq N$. Then $F(x([t]))=0$ and, by (8) and (13), $x(t) \leq x([t])$. Hence, $\lim _{t \rightarrow \infty} x(t)=-\infty$.

Our main results characterize eventually periodic solutions $x=x(t)$ of (2). Since $x(t)$ is generally not differentiable at integers $t=n$ but is always differentiable between integers, we restrict our attention to integral periods (see $[3,6])$. In this case, by (8), we have the following:

$x=x(t)$ is eventually periodic with positive integral period $p$ if and only if $x(n)(n=0,1, \ldots)$ is eventually periodic with period $p$.

Equation (2) is similar to recent models related to neural networks ([8-12]). We treat a generalized version of (6) as follows.

For real numbers $a, b, x_{0}$, and $\lambda>0$, define, for $n \geq 1$,

$$
x_{n}=a x_{n-1}-b F\left(x_{n-1}\right),
$$

where $\lambda$ and $F$ are given by (3).

Remark 2. Equation (18) is the difference equation (6) of a differential equation (2) with $x(0) \equiv x_{0}, \lambda \equiv \lambda$, and $A$ being arbitrary: if $A=0$, choose $B \equiv a-1$ and $C \equiv-b$. And if $A \neq 0$, then let $B \equiv\left(a-e^{A}\right) A /\left(e^{A}-1\right)$ and $C \equiv-b A /\left(e^{A}-1\right)$. The resulting solution $x=x(t)$ given by (8) satisfies (2) with $a^{*} \equiv a$ and $b^{*} \equiv b$.

The next result shows that we may henceforth assume that $a>1$ and $b>0$ in (18).

Proposition 3. Let $x_{n}$ be defined by (18) for real $a, b, x_{0}$, and $\lambda>0$, and let $x=x(t)$ satisfy (8), where $a^{*}$ and $b^{*}$ are given in (6). In the following, $j$ is an arbitrary positive integer.

(1) (a) Suppose that $0 \leq a \leq 1$. If $x_{k} \leq \lambda$, for some $k$, then $x_{k+j}=a^{j} x_{k}$. On the other hand, if $x_{k}>\lambda$, for all $k$, then

$$
x_{j}= \begin{cases}a^{j}\left(x_{0}-\frac{b}{a-1}\right)+\frac{b}{a-1}, & \text { if } a \neq 1 \\ x_{0}-j b, & \text { if } a=1(b \leq 0) .\end{cases}
$$

(b) In particular, assume that $0 \leq a^{*} \leq 1$. If $x(k) \leq \lambda$, for some nonnegative integer $k$, then, for all $t^{\prime}$ in $[k, k+1)$, one has that $x\left(t^{\prime}+j\right)=a^{* j} x\left(t^{\prime}\right)$. On the other hand, if $x(k)>\lambda$, for all nonnegative integers $k$, then, for all $t^{\prime}$ in $[0,1)$,

$$
\begin{aligned}
& x\left(t^{\prime}+j\right) \\
& = \begin{cases}a^{* j}\left(x\left(t^{\prime}\right)-\frac{b^{*}}{a^{*}-1}\right)+\frac{b^{*}}{a^{*}-1}, & \text { if } a^{*} \neq 1 \\
x\left(t^{\prime}\right)-j b^{*}, & \text { if } a^{*}=1\left(b^{*} \leq 0\right) .\end{cases}
\end{aligned}
$$

(2) (a) Suppose that $a>1$ and $b \leq 0$. If $x_{0} \leq 0$, then $x_{j}=a^{j} x_{0}$. If $x_{0}>0$, then there exists $k \geq 1$ such that $x_{k+j}=$ $a^{j}\left(x_{k}-b /(a-1)\right)+b /(a-1)$.

(b) In particular, assume that $a^{*}>1$ and $b^{*} \leq 0$. If $x(0) \leq$ 0 , then $x\left(t^{\prime}+j\right)=a^{* j} x\left(t^{\prime}\right)$ for all $t^{\prime}$ in $[0,1)$. If $x(0)>0$, then there exists a positive integer $k$ such that $x\left(t^{\prime}+j\right)=a^{* j}\left(x\left(t^{\prime}\right)-\right.$ $\left.b^{*} /\left(a^{*}-1\right)\right)+b^{*} /\left(a^{*}-1\right)$ for all $t^{\prime}$ in $[k, k+1)$.

(3) (a) Suppose that $a<0$. Either $x_{n}$ is oscillatory or there exists $k \geq 0$ such that $x_{k+j}=a^{j}\left(x_{k}-b /(a-1)\right)+b /(a-1)$.

(b) In particular, assume that $a^{*}<0$. Either $x=x(t)$ is oscillatory or there exists an integer $k \geq 0$ such that $x\left(t^{\prime}+j\right)=$ $a^{* j}\left(x\left(t^{\prime}\right)-b^{*} /\left(a^{*}-1\right)\right)+b^{*} /\left(a^{*}-1\right)$ for all $t^{\prime}$ in $[k, k+1)$.

Proof. (1a) Suppose that $0 \leq a \leq 1$. If $x_{k} \leq \lambda$, for some $k$, then either $0 \leq x_{k+1}=a x_{k} \leq x_{k} \leq \lambda$ or $x_{k+1}=a x_{k} \leq 0<\lambda$. Hence, the desired form of $x_{k+j}$ follows by induction in this case.

Assume that $x_{k}>\lambda$ for all $k$. Thus, if $a \neq 1$, then

$$
\begin{aligned}
x_{j} & =a x_{j-1}-b=a^{2} x_{j-2}-\frac{a^{2}-1}{a-1} b=\cdots \\
& =a^{j}\left(x_{0}-\frac{b}{a-1}\right)+\frac{b}{a-1} .
\end{aligned}
$$

And if $a=1$, then $x_{j}=x_{j-1}-b=x_{j-2}-2 b=\cdots=x_{0}-j b$. (Since $x_{j}>\lambda>0$, for all $j$, it follows that $b \leq 0$ in this case.)

(1b) Suppose that $0 \leq a^{*} \leq 1$. Let $x(k) \leq \lambda$ for some integer $k \geq 0$. By (a), $x(k+j)=a^{* j} x(k) \leq \lambda$ and $F(x(k+j))=$ 0 . Let $t^{\prime}$ be in $[k, k+1)$. Then $k+j \leq t^{\prime}+j<k+j+1$ and, by $(8)$,

$$
\begin{aligned}
x & \left(t^{\prime}+j\right) \\
& =\left(e^{A\left(t^{\prime}+j-\left[t^{\prime}+j\right]\right)}+B \int_{\left[t^{\prime}+j\right]}^{t^{\prime}+j} e^{A\left(t^{\prime}+j-s\right)} d s\right) x(k+j) \\
& =a^{* j}\left(e^{A\left(t^{\prime}-\left[t^{\prime}\right]\right)}+B \int_{\left[t^{\prime}\right]}^{t^{\prime}} e^{A\left(t^{\prime}-s\right)} d s\right) x(k) \\
& =a^{* j} x\left(t^{\prime}\right) .
\end{aligned}
$$

Next, assume that $x(k)>\lambda$ and thus $F(x(k))=1$ for all integers $k \geq 0$. Suppose first that $a^{*} \neq 1$ and $0 \leq t^{\prime}<1$. By (a), $x(j)=a^{* j}\left(x(0)-b^{*} /\left(a^{*}-1\right)\right)+b^{*} /\left(a^{*}-1\right)$. Since $j \leq t^{\prime}+j<j+1$ and $b^{*} /\left(a^{*}-1\right)=-C /(A+B)$,

$$
\begin{aligned}
x\left(t^{\prime}+j\right)= & a^{* j}\left(e^{A\left(t^{\prime}-\left[t^{\prime}\right]\right)}+B \int_{\left[t^{\prime}\right]}^{t^{\prime}} e^{A\left(t^{\prime}-s\right)} d s\right) \\
& \cdot\left(x(0)-\frac{b^{*}}{a^{*}-1}\right)+\frac{b^{*}}{a^{*}-1}
\end{aligned}
$$

as in the proof of Proposition 1(1). Therefore, $x\left(t^{\prime}+j\right)=$ $a^{* j}\left(x\left(t^{\prime}\right)-b^{*} /\left(a^{*}-1\right)\right)+b^{*} /\left(a^{*}-1\right)$. 
Finally suppose that $a^{*}=1$. Thus $B=-A$ and, by (a), $x(j)=x(0)-j b^{*}>\lambda$ and $b^{*} \leq 0$. As above, for $0 \leq t^{\prime}<1$, we have that $j \leq t^{\prime}+j<j+1$ and

$$
x\left(t^{\prime}+j\right)=x(j)+C \int_{\left[t^{\prime}\right]}^{t^{\prime}} e^{A\left(t^{\prime}-s\right)} d s=x\left(t^{\prime}\right)-j b^{*} .
$$

(2a) Suppose that $a>1$ and $b \leq 0$. If $x_{0} \leq 0(<\lambda)$, then clearly $x_{j}=a^{j} x_{0}$. Assume that $x_{0}>0$. There exists a positive integer $k$ such that $x_{k-1}=a^{k-1} x_{0} \leq \lambda<x_{k}=a^{k} x_{0}$. Thus, $x_{k+1}=a^{k+1} x_{0}-b=a x_{k}-b>x_{k}>\lambda$ since $a>1, x_{k}>0$, and $b \leq 0$. As in the proof of (1a), $x_{k+j}=a^{j} x_{k}-\left(\left(a^{j}-1\right) /(a-1)\right) b$ $(>\lambda)$.

(2b) Suppose that $a^{*}>1$ and $b^{*} \leq 0$. If $x(0) \leq 0$, then $x(j)=a^{* j} x(0)<\lambda$ by $(2 \mathrm{a})$; and hence the result follows as in the proof of (1b).

Assume that $x(0)>0$. By $(2 \mathrm{a})$, there exists a positive integer $k$ such that $x(k)>\lambda$ and $x(k+j)=a^{* j}(x(k)-$ $\left.b^{*} /\left(a^{*}-1\right)\right)+b^{*} /\left(a^{*}-1\right)>\lambda$. Let $k \leq t^{\prime}<k+1$ so that $k+j \leq t^{\prime}+j<k+j+1$. Then, by (8),

$$
\begin{gathered}
x\left(t^{\prime}+j\right)=a^{* j}\left(e^{A\left(t^{\prime}-\left[t^{\prime}\right]\right)}+B \int_{\left[t^{\prime}\right]}^{t^{\prime}} e^{A\left(t^{\prime}-s\right)} d s\right) \\
\cdot\left(x(k)-\frac{b^{*}}{a^{*}-1}\right)+\frac{b^{*}}{a^{*}-1} \\
=a^{* j}\left(x\left(t^{\prime}\right)-\frac{b^{*}}{a^{*}-1}\right)+\frac{b^{*}}{a^{*}-1}
\end{gathered}
$$

as in the proof of (1b).

(3a) Suppose that $a<0$. One of the following must be true:

(i) For every integer $k \geq 0$, there exists an integer $n_{k} \geq k$ such that $x_{n_{k}} \leq \lambda$.

(ii) There exists an integer $k \geq 0$ such that $x_{n}>\lambda$ for all $n \geq k$.

Assume that (i) holds. If $x_{j}=0$, for some $j$, then $x_{n}$ is oscillatory with stationary state 0 ; thus we further assume that $x_{n}$ has no zero terms. Therefore, there exists a subsequence of $x_{n}$ with alternating signs.

We may choose $x_{n_{0}}>0$ as follows. If $x_{0}>0$, then let $x_{n_{0}} \equiv x_{0}$. If $x_{0}<0(<\lambda)$, then let $x_{n_{0}} \equiv x_{1}=a x_{0}>0$.

Next choose $x_{n_{1}}<0$, where $n_{1}>n_{0}$ : by (i), there exists $n_{1}^{\prime} \geq n_{0}$ such that $x_{n_{1}^{\prime}} \leq \lambda$. If $x_{n_{1}^{\prime}}<0$, then $n_{1}^{\prime}>n_{0}$ so let $n_{1} \equiv n_{1}^{\prime}$. If $x_{n_{1}^{\prime}}>0$, then $x_{n_{1}^{\prime}+1}=a x_{n_{1}^{\prime}}<0$ and $n_{1}^{\prime}+1>n_{0}$; thus, in this case, let $n_{1} \equiv n_{1}^{\prime}+1$.

Since $x_{n_{1}}<0<\lambda$, we have $x_{n_{2}} \equiv x_{n_{1}+1}=a x_{n_{1}}>0$ and $n_{2}>n_{1}$. By induction, a sequence $x_{n_{k}}$ is constructed such that $n_{k+1}>n_{k}$ and $x_{n_{k}} x_{n_{k+1}}<0$ for all $k$. Thus $x_{n}$ is oscillatory.

On the other hand, if (ii) holds, then, as in the proof of (1a), there exists $k \geq 0$ such that $x_{k+j}=a^{j}\left(x_{k}-b /(a-1)\right)+$ $b /(a-1)$.

(3b) Suppose that $a^{*}<0$. By (3a), either $x(n)$ is oscillatory (and hence $x=x(t)$ is oscillatory) or $x(k+j)=a^{* j}(x(k)-$ $\left.b^{*} /\left(a^{*}-1\right)\right)+b^{*} /\left(a^{*}-1\right)>\lambda$ (with $\left.x(k)>\lambda\right)$ for some integer $k \geq 0$. The desired result follows in the latter case as in the proof of $(2 b)$.
Thus we assume that $a^{*}>1$ and $b^{*}>0$; and therefore Proposition 1 applies to the resulting solution $x=x(t)$ of (2). It will follow from the third section that if $\lambda$ is outside the interval $\left(b^{*} / a^{* 2}, b^{*} /\left(a^{*}-1\right)-b^{*} / a^{* 2}\right)$, then $x(t)$ is either eventually constant or unbounded. Moreover, if $a^{*} \geq 2$ and $\lambda$ is in $\left[b^{*} /\left(a^{*}\left(a^{*}-1\right)\right), b^{*} / a^{*}\right]$, then, by the fourth section, all initial values $x(0)$ are derived such that $x(t)$ is eventually periodic; and more generally, for any $\lambda>0$, the eventually periodic solutions of (2) are the bounded solutions with these initializations $x(0)$.

Remark 4. For integers $a, b, x_{0}$, and $\lambda>0$, periodic solutions of difference equation (18) were used in [13] to determine the real eigenvalues of certain arbitrarily large, sparse matrices.

\section{Unbounded Solutions}

Let $x_{n}$ satisfy (18), where $a>1$ and $b>0$. If $x_{n}$ is unbounded, then $x_{n}$ is eventually geometric: we define

$$
\begin{aligned}
& f_{-}(i, j) \equiv a^{j} x_{i} \quad \text { whenever } x_{i} \leq 0 \\
& f_{+}(i, j) \equiv a^{j}\left(x_{i}-\frac{b}{a-1}\right)+\frac{b}{a-1} \\
& \text { when } x_{i} \geq \frac{b}{a-1} .
\end{aligned}
$$

The following result shows that we may assume

$$
\begin{aligned}
& 0<x_{0}<\frac{b}{a-1}, \\
& \lambda<\frac{b}{a-1}
\end{aligned}
$$

since otherwise there exists $i \geq 0$ such that $x_{i+j}=f_{ \pm}(i, j)$ for all $j$.

Lemma 5. Assume that $x_{n}$ is defined by (18), where $a>1$ and $b>0$.

(1) If $x_{n} \leq \lambda$, for all $n \geq i \geq 0$, then $x_{i+j}=f_{-}(i, j)$ for all $j \geq 0$. In particular, if $x_{i} \leq 0$, for some $i$, then $x_{i+j}=$ $f_{-}(i, j)$ for all $j$.

(2) If $x_{n}>\lambda$, for all $n \geq i \geq 0$, then $x_{i+j}=f_{+}(i, j)$ for all $j \geq 0$. In particular, if $x_{i} \geq b /(a-1)>\lambda$, for some $i$, then $x_{i+j}=f_{+}(i, j)$ for all $j$.

(3) If $x_{i}>\lambda \geq b /(a-1)$, for some $i$, then $x_{i+j}=f_{+}(i, j)$ for all $j \geq 0$. In particular, if either $0<x_{i} \leq b /(a-1) \leq \lambda$ or $b /(a-1) \leq x_{i} \leq \lambda$, for some $i$, then there exists $k>i$ such that $x_{k+j}=f_{+}(k, j)$ for all $j$.

Proof. (1) Assume that $x_{n} \leq \lambda$ for all $n \geq i$. Then $x_{i+j}=a^{j} x_{i}$ for all $j \geq 0$. If $x_{i}>0$, then, since $a>1$, it follows that $x_{n}$ is not bounded above which contradicts our hypothesis. Thus $x_{i} \leq 0$ and $x_{i+j}=f_{-}(i, j)$. 
If $x_{i} \leq 0(<\lambda)$, for some $i$, then clearly $x_{n} \leq \lambda$ for all $n \geq i$.

(2) Suppose that $x_{n}>\lambda$ for all $n \geq i$. Then

$$
\begin{aligned}
& x_{i+1}=a x_{i}-b=a\left(x_{i}-\frac{b}{a-1}\right)+\frac{b}{a-1}, \\
& x_{i+2}=a x_{i+1}-b=a^{2}\left(x_{i}-\frac{b}{a-1}\right)+\frac{b}{a-1}
\end{aligned}
$$

and, by induction, for all $j$,

$$
x_{i+j}=a^{j}\left(x_{i}-\frac{b}{a-1}\right)+\frac{b}{a-1} .
$$

If $x_{i}<b /(a-1)$, then, since $a>1, x_{n}$ is not bounded below which is contrary to our hypothesis. Thus $x_{i} \geq b /(a-1)$ and $x_{i+j}=f_{+}(i, j)$ for all $j$.

Assume that $x_{i} \geq b /(a-1)>\lambda$ for some $i$. Then $x_{i}=$ $f_{+}(i, 0)>\lambda$ since $x_{i} \geq b /(a-1)$, and

$$
x_{i+1}=a x_{i}-b \geq a\left(\frac{b}{a-1}\right)-b=\frac{b}{a-1}>\lambda,
$$

where $x_{i+1}=f_{+}(i, 1)$ as above.

Similarly, by induction,

$$
x_{i+j}=a x_{i+j-1}-b \geq \frac{b}{a-1}>\lambda
$$

and $x_{i+j}=f_{+}(i, j)$ for all $j$.

(3) Assume that $x_{i}>\lambda \geq b /(a-1)$ for some $i$. Then $x_{i}=f_{+}(i, 0)>\lambda$ and

$$
x_{i+1}=a x_{i}-b>a \lambda-(a-1) \lambda=\lambda
$$

where $x_{i+1}=f_{+}(i, 1)$. By induction, $x_{i+j}=a x_{i+j-1}-b>\lambda$ and $x_{i+j}=f_{+}(i, j)$ for all $j$.

Suppose that $0<x_{i} \leq b /(a-1) \leq \lambda$ for some $i$. There exists $k>i$ such that

$$
x_{k-1}=a^{k-1-i} x_{i} \leq \lambda<x_{k}=a^{k-i} x_{i} .
$$

Thus, by the general case, $x_{k+j}=f_{+}(k, j)$ for all $j$.

Similarly, if $b /(a-1) \leq x_{i} \leq \lambda$, then there is $k>i$ such that $x_{k+j}=f_{+}(k, j)$ for all $j$.

The next result is central to our analysis.

Lemma 6. Let $x_{n}$ be given by (18), where $a>1, b>0$, and $0<\lambda, x_{0}<b /(a-1)$.

(1) If $\lambda \leq b / a(a-1)$, then $x_{n} \leq b /(a-1)$ for all $n$. In particular, if $\lambda<b / a(a-1)$, then $x_{n}<b /(a-1)$ for all $n$.

(2) Ifb/a $(a-1) \leq x_{0} \leq \lambda$, then $x_{1+j}=f_{+}(1, j)$ for all $j \geq 0$. In this case, $x_{0}=b / a(a-1)$ if and only if $x_{1}=b /(a-1)$.

(3) If $\lambda \geq b / a$, then $x_{n}>0$ for all $n$.

(4) If $b / a \geq x_{0}>\lambda$, then $x_{1+j}=f_{-}(1, j)$ for all $j \geq 0$. In this case, $x_{0}=b / a$ if and only if $x_{1}=0$.
Proof. (1) Assume $\lambda \leq b / a(a-1)$ and $x_{0}<b /(a-1)$.

If $x_{0} \leq \lambda$, then $x_{1}=a x_{0} \leq a \lambda \leq b /(a-1)$. And if $x_{0}>\lambda$, then $x_{1}=a x_{0}-b<a(b /(a-1))-b=b /(a-1)$. Thus $x_{1} \leq b /(a-1)$ in both cases, and, similarly, by induction, $x_{n} \leq b /(a-1)$ for all $n$.

If $\lambda<b / a(a-1)$, then $x_{n}<b /(a-1)$ for all $n$ by the above argument.

(2) Suppose that $b / a(a-1) \leq x_{0} \leq \lambda$. Then $x_{1}=a x_{0} \geq$ $b /(a-1)>\lambda$ and $x_{1+j}=f_{+}(1, j)$ for all $j$ by Lemma 5(2).

If $b / a(a-1)=x_{0} \leq \lambda$, then $x_{1}=a x_{0}=b /(a-1)$. Conversely, if $b / a(a-1) \leq x_{0} \leq \lambda$ and $x_{1}=b /(a-1)$, then $x_{1}=a x_{0}$ so $x_{0}=b / a(a-1)$.

(3) Assume $\lambda \geq b / a$. If $x_{0} \leq \lambda$, then $x_{1}=a x_{0}>0$. And if $x_{0}>\lambda$, then $x_{1}=a x_{0}-b>a \lambda-b \geq 0$. Thus $x_{1}>0$ and, by induction, $x_{n}>0$ for all $n$.

(4) Suppose that $b / a \geq x_{0}>\lambda$. Then $x_{1}=a x_{0}-b \leq$ $a(b / a)-b=0$ and $x_{1+j}=f_{-}(1, j)$ for all $j$ by Lemma 5(1).

Clearly, $x_{0}=b / a$ if and only if $x_{1}=0$ in this case.

Remark 7. Let $x_{n}$ be defined as in Lemma 6. As in the proof of Lemma 5(3), if $x_{0} \leq \lambda$, then there is a unique positive integer $i$ such that $a^{i-1} x_{0} \leq \lambda<a^{i} x_{0}$. In this case, $x_{k}=a^{k} x_{0}$ for $k=1, \ldots, i$; and if $x_{i} \leq b / a$, then $x_{i+1+j}=a^{j} x_{i+1}$ for all $j$ by Lemma 6(4) since $x_{i}>\lambda$.

Similarly, if $x_{0}>\lambda$, then there is a unique positive integer $i$ such that

$$
\begin{aligned}
0 & <a^{i-1}\left(\frac{b}{a-1}-x_{0}\right)<\frac{b}{a-1}-\lambda \\
& \leq a^{i}\left(\frac{b}{a-1}-x_{0}\right) .
\end{aligned}
$$

In this case, writing $x_{0}=b /(a-1)-\left(b /(a-1)-x_{0}\right)>\lambda$, we have that

$$
x_{k}=a x_{k-1}-b=\frac{b}{a-1}-a^{k}\left(\frac{b}{a-1}-x_{0}\right)>\lambda
$$

for $k=1, \ldots, i$; and if $x_{i} \geq b / a(a-1)$, then

$$
x_{i+1+j}=\frac{b}{a-1}-a^{j}\left(\frac{b}{a-1}-x_{i+1}\right)
$$

for all $j$ by Lemma 6(2) since $x_{i} \leq \lambda$.

Example 8. Let $a \geq 2$ and let $b>0$ and assume that $b / a(a-$ 1) $\leq \lambda \leq b / a$. Let $x_{0}$ be the weighted average

$$
x_{0} \equiv \frac{(2 a-1)\left(b / a^{2}\right)+b / a(a-1)}{2 a} .
$$

Then $x_{0}<\lambda$ and

$$
x_{1}=a x_{0}=\frac{((2 a-1)(a-1)+a) b}{2 a^{2}(a-1)}>\frac{b}{a} \geq \lambda \text {. }
$$

Thus

$$
x_{2}=a x_{1}-b=\frac{b}{2 a(a-1)}<\frac{b}{a(a-1)} \leq \lambda
$$

and $x_{3}=a x_{2}=b / 2(a-1)$ is the midpoint of $[b / a(a-1), b / a]$.

If $x_{3} \leq \lambda$, then $x_{4+j}=f_{+}(4, j)$ for all $j$ by Lemma $6(2)$. And if $x_{3}>\lambda$, then $x_{4+j}=f_{-}(4, j)$ for all $j$ by Lemma $6(4)$. 


\section{Bounded Solutions}

By Lemma 5, solutions $x_{n}$ of (18) such that $a>1, b>0$, and $0<\lambda, x_{0}<b /(a-1)$ are bounded only when $0 \leq x_{n} \leq$ $b /(a-1)$ for all $n$. In particular, by Lemma 6 , if $a \leq 2$ and $b / a \leq \lambda \leq b / a(a-1)$, then $x_{n}$ is bounded. It is possible that $x_{n}$ is bounded but not eventually periodic.

Example 9. Assume that $1<a=p / q<2$, where $p$ and $q$ are odd and even integers, respectively; $b>0$ and $b / a \leq \lambda \leq$ $b / a(a-1)$. If $x_{0} \equiv(b /(a-1))(r / s)$, where $r$ and $s$ are odd and even integers, respectively, such that $r<s$, then the solution $x_{n}$ is bounded but not eventually periodic: by Lemma $6,0<$ $x_{n} \leq b /(a-1)$ for all $n$. Note that

$$
\begin{aligned}
a x_{0} & =\frac{b}{a-1}\left(\frac{p r}{q s}\right), \\
a x_{0}-b & =\frac{b}{a-1}\left(\frac{p r-(p-q) s}{q s}\right)
\end{aligned}
$$

so that $x_{1}=(b /(a-1))\left(p_{1} / q s\right)$, where $p_{1}$ is odd and $q s$ is even.

Similarly, by induction, for all $n \geq 1, x_{n}=(b /(a-$ 1)) $\left(p_{n} / q^{n} s\right)$, where $p_{n}$ is odd and $q^{n} s$ is even. It follows that $x_{n}$ is not eventually periodic since if $x_{m}=x_{m+n}$ for some $m \geq 0$ and $n \geq 1$, then $p_{m+n}=q^{n} p_{m}$ is even.

We now classify the types of solutions that may be bounded. Our results will be stated in terms of the decomposition of $(0, b /(a-1))$ into the disjoint union of the intervals $I_{1}=\left(0, b / a^{2}\right], I_{2}=\left(b / a^{2}, \min \{b / a, b / a(a-\right.$ $1)\}), I_{3}=[\min \{b / a, b / a(a-1)\}, \max \{b / a, b / a(a-1)\}], I_{4}=$ $\left(\max \{b / a, b / a(a-1)\}, b /(a-1)-b / a^{2}\right)$, and $I_{5}=[b /(a-1)-$ $\left.b / a^{2}, b /(a-1)\right)$.

Definition 10. Let $a>1, b>0$, and $0<\lambda, x_{0}<b /(a-1)$. A solution $x_{n}$ defined by (18) is of

(i) type A if $\lambda$ and $x_{k}$ are in $I_{2}$ for some $k$ such that $x_{k} \leq \lambda$,

(ii) type B if $\lambda$ and $x_{k}$ are in $I_{4}$ for some $k$ such that $x_{k}>\lambda$,

(iii) type $\mathrm{C}$ if $\lambda$ is in $I_{3}$ and either $a \geq 2$ such that $x_{k}$ is in $I_{2} \cup I_{4}$ for some $k$ or $a<2$.

The unbounded solution in Example 8 is of type C.

Let $x_{n}$ satisfy (18) as in Definition 10 . Since $\lambda<b /(a-1)$, we have that $c$ is a stationary state of $x_{n}$ if and only if $x_{i}=c=0$ or $x_{i}=c=b /(a-1)$ for some $i$, in which case $x_{i+j}=c$ for all $j \geq 0$ : if $x_{i}=0(<\lambda)$ or $x_{i}=b /(a-1)(>\lambda)$, then clearly $x_{i+1}=x_{i}=c$. Conversely, suppose that $x_{i+1}=x_{i}=c$ for some $i$. If $c \leq \lambda$, then $c=x_{i+1}=a x_{i}=a c$ so $c=0$ since $a>1$. And if $c>\lambda$, then $c=x_{i+1}=a x_{i}-b=a c-b$ so $c=b /(a-1)$.

The solution $x_{n}$ is eventually periodic if there are integers $m \geq 0$ and $p \geq 1$ such that $x_{m}=x_{m+p}$ (and thus, by (18), $x_{m+j}=x_{m+p+j}$ for all $j \geq 0$ ). The following eventually periodic solution is either of type A or type C.
Example 11. Let $a>1$ and let $b>0$ and let $p \geq 2$ be an integer such that $a^{p} \geq a /(a-1)$. (If $a \geq 2$, then $p \geq 2$ is arbitrary.) Choose $\lambda$ such that

$$
\frac{b}{a^{2}} \leq \frac{b / a^{2}}{1-1 / a^{p}}=\frac{b a^{p-2}}{a^{p}-1} \leq \lambda \leq \frac{b}{a}
$$

and, for any integer $m \geq 0$, let

$$
x_{0} \equiv \frac{b}{a^{m}\left(a^{p}-1\right)} \text {. }
$$

Then $x_{m}=x_{m+p}: x_{0} \leq b a^{p-2} /\left(a^{p}-1\right) \leq \lambda$ so $x_{1}=$ $b /\left(a^{m-1}\left(a^{p}-1\right)\right)$. Similarly, $x_{m}=a^{m} x_{0}=b /\left(a^{p}-1\right) \leq$ $b a^{p-2} /\left(a^{p}-1\right) \leq \lambda$ and, for $k=1, \ldots, p-2, x_{m+k}=b a^{k} /\left(a^{p}-\right.$ $1) \leq b a^{p-2} /\left(a^{p}-1\right) \leq \lambda$. Thus $x_{m+(p-1)}=b a^{p-1} /\left(a^{p}-1\right)>$ $b / a \geq \lambda$ and

$$
x_{m+p}=a x_{m+(p-1)}-b=\frac{b}{a^{p}-1}=x_{m} .
$$

Note that if $a \geq 2$, then, since $b / a^{2} \leq x_{m+p-2} \leq b / a(a-$ $1) \leq b / a$, it follows that $x_{n}$ is of type A whenever $\lambda$ is in $I_{2}$ (and $\left.x_{m+p-2} \leq \lambda\right)$ and is of type $\mathrm{C}$ when $\lambda$ is in $I_{3}$. If $a<2$, then $x_{n}$ is of type A since $x_{m+p-2}$ and $\lambda$ are in $I_{2}$ and $x_{m+p-2} \leq \lambda$. The following slight modification is eventually periodic of type $\mathrm{C}$ when $a<2$.

Example 12. Let $1<a<2$ and let $b>0$ and let $p \geq 2$ be an integer such that

$$
a^{p}>\max \left\{\frac{a}{a-1}, \frac{1}{2-a}\right\}
$$

and let $\lambda$ in $I_{3}$ satisfy

$$
\frac{b}{a^{2}}<\frac{b a^{p-2}}{a^{p}-1}<\frac{b}{a}<\lambda<\frac{b a^{p-1}}{a^{p}-1}<\frac{b}{a(a-1)} .
$$

Define

$$
x_{0} \equiv \frac{b a^{q}}{a^{m}\left(a^{p}-1\right)}
$$

for integers $m \geq 0$ and $q$ such that $0 \leq q \leq p-2$. Then $x_{0} \leq b a^{p^{-2}} /\left(a^{p}-1\right)<\lambda$ and, as in Example 11,

$$
\begin{gathered}
x_{m}=\frac{b a^{q}}{a^{p}-1} \leq \frac{b a^{p-2}}{a^{p}-1}<\lambda, \\
x_{m+p-q-2}=a^{p-q-2} x_{m}=\frac{b a^{p-2}}{a^{p}-1}<\lambda .
\end{gathered}
$$

Therefore, $x_{m+p-q-1}=b a^{p-1} /\left(a^{p}-1\right)>\lambda$,

$$
\begin{gathered}
x_{m+p-q}=a x_{m+p-q-1}-b=\frac{b}{a^{p}-1}<\lambda, \\
x_{m+p}=a^{q} x_{m+p-q}=\frac{b a^{q}}{a^{p}-1}=x_{m} .
\end{gathered}
$$


Note that, for an element $x_{0}$ in $I_{1}$, there exists an integer $k \geq 2$ such that

$$
\frac{b}{a^{k+1}}<x_{0} \leq \frac{b}{a^{k}}
$$

Similarly, for $x_{0}$ in $I_{5}$, there exists an integer $k \geq 2$ such that

$$
\frac{b}{a-1}-\frac{b}{a^{k}} \leq x_{0}<\frac{b}{a-1}-\frac{b}{a^{k+1}} \text {. }
$$

Inequalities (49) and (50) will be used repeatedly in the next result.

In seeking bounded solutions without stationary states as in Examples 11 and 12, we may further assume that $\lambda$ is in $I_{2} U$ $I_{3} \cup I_{4}$ by the next result.

Theorem 13. Let $x_{n}$ be a solution of (18) such that $a>1, b>0$, and $0<\lambda, x_{0}<b /(a-1)$. Then $x_{0}$ fits one and only one of the following cases.

(1) Let $\lambda$ be in $I_{1}$.

(a) If $x_{0} \leq \lambda$ and $k \geq 2$ is given by (49), then $x_{i+j}=$ $f_{-}(i, j)$ for all $j \geq 0$, where $i$ is the first integer in $[2, k]$ such that $a^{i-1} x_{0}>\lambda$; that is, $x_{i-1}>\lambda$. In this case, if $\lambda=b / a^{k}$, then $i=2$; and $x_{2}<0$ when $k>2$.

(b) If $\lambda<x_{0} \leq b / a$, then $x_{1+j}=f_{-}(1, j)$ for all $j \geq 0$.

(2) Let $\lambda$ be in $I_{2}$.

(a) If $x_{0}$ is in $I_{1}$ and $k \geq 2$ is given by (49), then $x_{k-1}=a^{k-1} x_{0}$ and one has the following.

(i) If $b / a^{2}<x_{k-1} \leq \lambda$, then $x_{n}$ is of type A.

(ii) If $\lambda<x_{k-1} \leq b / a$, then $x_{k+j}=f_{-}(k, j)$ for all $j$.

(b)

(i) If $b / a^{2}<x_{0} \leq \lambda$, then $x_{n}$ is of type $A$.

(ii) If $\lambda<x_{0} \leq b / a$, then $x_{1+j}=f_{-}(1, j)$ for all $j$.

(3) Let $\lambda$ be in $I_{1} \cup I_{2}$.

(a) Let $x_{0} \neq b / a$ be in $I_{3}$ and let $\phi=(1+\sqrt{5}) / 2$ be the golden ratio with the property that $\phi(\phi-1)=1$.

(i) If $\phi \leq a<2$, then either (1) or (2) holds for the sequence $x_{n}(n \geq 1)$ with initial term $x_{1}=a x_{0}-b$.

(ii) If $a<\phi$ and $r$ is the least positive integer such that $a^{r}(a-1) \geq 1$, then there exists an integer $s$ in $[1, r]$ such that $x_{s}=b /(a-1)-$ $a^{s}\left(b /(a-1)-x_{0}\right)$ is in $I_{1} \cup I_{2} \cup\{b / a\}$; and therefore (1) or (2) applies to $x_{n}(n \geq s)$.

(b) Let $x_{0}$ be in $I_{4}$.

(i) If $a \geq 2$, then $x_{1}=a x_{0}-b$ is in $I_{1} \cup I_{2}$ and $x_{n}(n \geq 1)$ satisfies either (1) or (2). (ii) If $a<2$, then $x_{1}=a x_{0}-b \neq b / a(a-1)$ is in $I_{1} \cup I_{2} \cup I_{3}$ and $x_{n}(n \geq 1)$ satisfies (1), (2), or (3a).

(c) Let $x_{0}$ be in $I_{5}$ and let $k \geq 2$ be given by (50). Then $x_{k}=b /(a-1)-a^{k}\left(b /(a-1)-x_{0}\right)$ and one has the following.

(i) If $a \geq 2$, then either $x_{k} \leq 0$ (and $x_{k+j}=$ $f_{-}(k, j)$, for all $\left.j\right)$, or $x_{k}$ is in $I_{1} \cup I_{2}$ and $x_{n}(n \geq k)$ satisfies (1) or (2).

(ii) If $a<2$, then $x_{k} \neq b / a(a-1)$ is in $I_{1} \cup I_{2} \cup I_{3}$ and $x_{n}(n \geq k)$ satisfies (1), (2), or (3a).

(4) Let $\lambda$ be in $I_{3}$. By Definition 10, if either $a<2$ or $a \geq 2$ and $I_{2} \cup I_{4}$ contains a term of the sequence $x_{n}$, then $x_{n}$ is of type $C$. Therefore, suppose that $a \geq 2$ and $x_{0}$ is in $I_{1} \cup I_{3} \cup I_{5}$

(a) Let $x_{0}$ be in $I_{1}$ and let $k \geq 2$ be given by (49). Then $x_{k-1}=a^{k-1} x_{0}$ is in $I_{2} \cup I_{3}$. If $x_{k-1}$ is in $I_{2}$, then $x_{n}$ is of type C. Let $x_{k-1}$ be in $I_{3}$. Then

(i) $x_{k+j}=f_{+}(k, j)$ for all $j \geq 0$ whenever $b / a(a-1) \leq x_{k-1} \leq \lambda$;

(ii) $x_{k+j}=f_{-}(k, j)$ for all $j \geq 0$ when $\lambda<$ $x_{k-1} \leq b / a$.

(b) Let $x_{0}$ be in $I_{3}$.

(i) If $x_{0} \leq \lambda$, then $x_{1+j}=f_{+}(1, j)$ for all $j$.

(ii) If $x_{0}>\lambda$, then $x_{1+j}=f_{-}(1, j)$ for all $j$.

(c) Let $x_{0}$ be in $I_{5}$ and let $k \geq 2$ be given by (50). Then $x_{k-1}=b /(a-1)-a^{k-1}\left(b /(a-1)-x_{0}\right)$ is in $I_{3} \cup I_{4}$. Thus, if $x_{k-1}$ is in $I_{4}$, then $x_{n}$ is of type $C$. Let $x_{k-1}$ be in $I_{3}$. Then

(i) $x_{k+j}=f_{+}(k, j)$ for all $j \geq 0$ whenever $b / a(a-1) \leq x_{k-1} \leq \lambda$;

(ii) $x_{k+j}=f_{-}(k, j)$ for all $j \geq 0$ when $\lambda<$ $x_{k-1} \leq b / a$.

(5) Let $\lambda$ be in $I_{4}$.

(a) If $x_{0}$ is in $I_{5}$ and $k \geq 2$ is given by (50), then $x_{k-1}=b /(a-1)-a^{k-1}\left(b /(a-1)-x_{0}\right)$ and one has the following.

(i) If $b / a(a-1) \leq x_{k-1} \leq \lambda$, then $x_{k+j}=$ $f_{+}(k, j)$ for all $j$.

(ii) If $\lambda<x_{k-1}<b /(a-1)-b / a^{2}$, then $x_{n}$ is of type $B$.

(b)

(i) If $b / a(a-1) \leq x_{0} \leq \lambda$, then $x_{1+j}=f_{+}(1, j)$ for all $j$.

(ii) If $\lambda<x_{0}<b /(a-1)-b / a^{2}$, then $x_{n}$ is of type $B$. 
(6) Let $\lambda$ be in $I_{5}$.

(a) If $x_{0}>\lambda$ and $k \geq 2$ is given by (50), then $x_{i+j}=$ $f_{+}(i, j)$ for all $j \geq 0$, where $i$ is the first integer in $[2, k]$ such that $a^{i-1}\left(b /(a-1)-x_{0}\right) \geq b /(a-1)-\lambda$; that is, $x_{i-1} \leq \lambda$. In this case, if $\lambda=b /(a-1)-$ $b / a^{k}$, then $i=2$ and $x_{2}>b /(a-1)$.

(b) If $b / a(a-1) \leq x_{0} \leq \lambda$, then $x_{1+j}=f_{+}(1, j)$ for all $j \geq 0$.

(7) Let $\lambda$ be in $I_{4} \cup I_{5}$.

(a) Let $x_{0} \neq b / a(a-1)$ be in $I_{3}$.

(i) If $\phi \leq a<2$, then either (5) or (6) holds for the sequence $x_{n}(n \geq 1)$ with initial term $x_{1}=a x_{0}$.

(ii) If $a<\phi$ and $r$ is the least positive integer such that $a^{r}(a-1) \geq 1$, then there exists an integer $s$ in $[1, r]$ such that $x_{s}=a^{s} x_{0}$ is in $\{b / a(a-1)\} \cup I_{4} \cup I_{5}$ and thus (5) or (6) applies to $x_{n}(n \geq s)$.

(b) Let $x_{0}$ be in $I_{2}$.

(i) If $a \geq 2$, then $x_{1}=a x_{0}$ is in $I_{4} \cup I_{5}$ and $x_{n}(n \geq 1)$ satisfies either (5) or (6).

(ii) If $a<2$, then $x_{1}=a x_{0} \neq b / a$ is in $I_{3} \cup I_{4} \cup I_{5}$ and $x_{n}(n \geq 1)$ satisfies (5), (6), or (7a).

(c) Let $x_{0}$ be in $I_{1}$ and let $k \geq 2$ be given by (49). Then $x_{k}=a^{k} x_{0}$ and one has the following.

(i) If $a \geq 2$, then either $x_{k} \geq b /(a-1)$ (hence $x_{k+j}=f_{+}(k, j)$ for all $\left.j\right)$ or $x_{k}$ is in $I_{4} \cup I_{5}$ and $x_{n}(n \geq k)$ satisfies (5) or (6).

(ii) If $a<2$, then $x_{k} \neq b / a$ is in $I_{3} \cup I_{4} \cup I_{5}$ and $x_{n}(n \geq k)$ satisfies (5), (6), or (7a).

Proof. (1a) Assume $0<x_{0} \leq \lambda \leq b / a^{2}$. By (49), there exists an integer $k \geq 2$ such that

$$
\begin{gathered}
\frac{b}{a^{k+1}}<x_{0} \leq \frac{b}{a^{k}} \leq \frac{b}{a^{2}}, \\
\lambda \leq \frac{b}{a^{2}}<a^{k-1} x_{0} .
\end{gathered}
$$

Therefore, as in Remark 7, there is a smallest integer $i$ in $[2, k]$ such that

$$
x_{i-2}=a^{i-2} x_{0} \leq \lambda<x_{i-1}=a^{i-1} x_{0} .
$$

It follows that

$$
x_{i}=a x_{i-1}-b=a^{i} x_{0}-b \leq a^{k} x_{0}-b \leq b-b=0
$$

and $x_{i+j}=f_{-}(i, j)$ for all $j$ by Lemma 5(1).

Suppose that $\lambda=b / a^{k}$. Then $\lambda / a<x_{0} \leq \lambda$ and $\lambda<x_{1}=$ $a x_{0}$ so $i=2$. And if $k>2$, then

$$
x_{2}=a x_{1}-b=a^{2} x_{0}-b<0 \quad \text { since } x_{0} \leq \frac{b}{a^{k}}<\frac{b}{a^{2}} .
$$

(1b) Lemma 6(4).
(2) Let $\lambda$ be in $I_{2}$. By (49), if $x_{0}$ is in $I_{1}$, then

$$
\frac{b}{a^{k+1}}<x_{0} \leq \frac{b}{a^{k}} \leq \frac{b}{a^{2}}<\lambda \text {. }
$$

Therefore, $b / a^{2}<x_{k-1}=a^{k-1} x_{0}$ and (2) follows from Definition 10 and Lemma 6(4).

Note that, for any $\lambda$ in $I_{1} \cup I_{2}$, (1) and (2) cover the following cases:

(i) $x_{0}$ is in $I_{1} \cup I_{2} \cup I_{3}$ whenever $a \geq 2$.

(ii) $x_{0}$ is in $I_{1} \cup I_{2} \cup\{b / a\}$ when $a<2$.

(3) Let $\lambda$ be in $I_{1} \cup I_{2}$.

(a) Suppose that $x_{0} \neq b / a$ is in $I_{3}$ and $a<2$. Then $\lambda<$ $b / a<x_{0} \leq b / a(a-1)$ and

$$
\begin{aligned}
0 & =a\left(\frac{b}{a}\right)-b<x_{1}=a x_{0}-b \leq \frac{b}{a-1}-b \\
& <\frac{b}{a(a-1)} .
\end{aligned}
$$

(i) Suppose that $\phi \leq a<2$. Then $a(a-1) \geq 1$ so $b /(a-$ $1)-b \leq b / a$ since $b / a(a-1) \leq b$. Thus, by (56), $x_{1}$ is in $I_{1} \cup I_{2} \cup\{b / a\}$ and (1) or (2) applies to $x_{n}(n \geq 1)$.

(ii) On the other hand, assume that $a<\phi$ so that $a$ ( $a-$ 1) < 1. By (56), $x_{1}$ is in $I_{1} \cup I_{2} \cup I_{3}$. If $x_{1}$ is in $I_{1} \cup$ $I_{2} \cup\{b / a\}$, then (1) or (2) applies to $x_{n}(n \geq 1)$. And if $x_{1} \neq b / a$ is in $I_{3}$, then, since $x_{1} \leq b /(a-1)-b$ by (56), the above argument shows

$$
0<x_{2}=a x_{1}-b \leq \frac{b}{a-1}-a b<\frac{b}{a(a-1)}
$$

and $x_{2}$ is in $I_{1} \cup I_{2} \cup I_{3}$.

Note that, in the latter case, if $x_{2} \neq b / a$ is in $I_{3}$, then $\lambda<$ $b / a<x_{2} \leq b /(a-1)-a b$ so $a^{2}(a-1)<1$.

It follows by induction that, for every $j \geq 1$, either $x_{j}$ is in $I_{1} \cup I_{2} \cup\{b / a\}$ or $x_{j} \neq b / a$ is in $I_{3}$ and $a^{j}(a-1)<1$. Since $a>1$, there are least positive integers $r$ and $s \leq r$ such that $a^{r}(a-1) \geq 1$ and $x_{s}$ is in $I_{1} \cup I_{2} \cup\{b / a\}$. Since $x_{i}>\lambda$, for $i=0, \ldots, s-1$, the form of $x_{s}$ follows from Remark 7 .

(b) Let $x_{0}$ be in $I_{4}$.

(i) Let $a \geq 2$. Then $\lambda<b / a<x_{0}<b /(a-1)-b / a^{2}$,

$$
\begin{aligned}
0 & =a\left(\frac{b}{a}\right)-b<x_{1}=a x_{0}-b<a\left(\frac{b}{a-1}-\frac{b}{a^{2}}\right)-b \\
& =\frac{b}{a(a-1)},
\end{aligned}
$$

and $x_{1}$ is in $I_{1} \cup I_{2}$.

(ii) Let $a<2$. Then $\lambda<b / a(a-1)<x_{0}<b /(a-1)-b / a^{2}$,

$$
\begin{aligned}
0 & <a\left(\frac{b}{a(a-1)}\right)-b<x_{1}=a x_{0}-b \\
& <a\left(\frac{b}{a-1}-\frac{b}{a^{2}}\right)-b=\frac{b}{a(a-1)},
\end{aligned}
$$

and $x_{1} \neq b / a(a-1)$ is in $I_{1} \cup I_{2} \cup I_{3}$. 
(c) Let $x_{0}$ be in $I_{5}$ and let $k \geq 2$ be given by (50). Then

$$
\lambda<\frac{b}{a-1}-\frac{b}{a^{2}} \leq \frac{b}{a-1}-\frac{b}{a^{k}} \leq x_{0}<\frac{b}{a-1}-\frac{b}{a^{k+1}}
$$

so that, for $j=1, \ldots, k-2$,

$$
\begin{aligned}
\lambda & <\frac{b}{a-1}-\frac{b}{a^{2}} \leq \frac{b}{a-1}-\frac{b}{a^{k-j}} \leq x_{j}=a x_{j-1}-b \\
& <\frac{b}{a-1}-\frac{b}{a^{k-j+1}} .
\end{aligned}
$$

Hence,

$$
\begin{aligned}
\lambda & <\frac{b}{a(a-1)}=\frac{b}{a-1}-\frac{b}{a} \leq x_{k-1} \\
& =a x_{k-2}-b<\frac{b}{a-1}-\frac{b}{a^{2}}, \\
\frac{b}{a-1}-b & \leq x_{k}=a x_{k-1}-b<\frac{b}{a-1}-\frac{b}{a}=\frac{b}{a(a-1)},
\end{aligned}
$$

where $b /(a-1)-b>0$ when $a<2$. Therefore, (3c) follows as above.

Moreover, by Remark 7, $x_{k}=b /(a-1)-a^{k}\left(b /(a-1)-x_{0}\right)$.

(4) Assume that $\lambda$ is in $I_{3}$.

(a) Let $x_{0}$ be in $I_{1}$ and $a \geq 2$. Then $b / a(a-1) \leq \lambda \leq b / a$ and, as in the proof of (2), if $k$ is given by (49), then $b / a^{2}<$ $x_{k-1}=a^{k-1} x_{0} \leq b / a$. Thus (4a) follows from Definition 10 and Lemma 6((2), (4)).

(b) Let $x_{0}$ be in $I_{3}$ and $a \geq 2$ so that $b / a(a-1) \leq x_{0} \leq b / a$. Hence, (i) and (ii) follow from Lemma 6((2), (4)).

(c) Let $x_{0}$ be in $I_{5}$ and $a \geq 2$. As in the proof of (3c),

$$
\begin{aligned}
\frac{b}{a(a-1)} & \leq x_{k-1}=\frac{b}{a-1}-a^{k-1}\left(\frac{b}{a-1}-x_{0}\right) \\
& <\frac{b}{a-1}-\frac{b}{a^{2}} .
\end{aligned}
$$

Therefore, (4c) follows from Definition 10 and Lemma 6((2), (4)).

(5) Assume that $\lambda$ is in $I_{4}$.

(a) Let $x_{0}$ be in $I_{5}$. As above, (63) holds for $x_{k-1}$. Moreover,

$$
\max \left\{\frac{b}{a}, \frac{b}{a(a-1)}\right\}<\lambda<\frac{b}{a-1}-\frac{b}{a^{2}} .
$$

Thus (5a) follows from Lemma 6(2) and Definition 10.

(b) A direct consequence of Lemma 6(2) and Definition 10.

(6) Let $\lambda$ be in $I_{5}$.

(a) Assume that $b /(a-1)-b / a^{2} \leq \lambda<x_{0}<b /(a-1)$ and let $k \geq 2$ be the integer satisfying (50):

$$
\frac{b}{a-1}-\frac{b}{a^{k}} \leq x_{0}<\frac{b}{a-1}-\frac{b}{a^{k+1}} \text {. }
$$

Thus

$$
\frac{b}{a-1}-a^{k-1}\left(\frac{b}{a-1}-x_{0}\right)<\frac{b}{a-1}-\frac{b}{a^{2}} \leq \lambda
$$

and, by Remark 7, there is a smallest integer $i$ in $[2, k]$ such that $x_{i-1} \leq \lambda$ and

$$
\begin{aligned}
x_{i-1} & =\frac{b}{a-1}-a^{i-1}\left(\frac{b}{a-1}-x_{0}\right) \leq \lambda<x_{i-2} \\
& =\frac{b}{a-1}-a^{i-2}\left(\frac{b}{a-1}-x_{0}\right) .
\end{aligned}
$$

It follows that

$$
x_{i}=a x_{i-1}=\frac{a b}{a-1}-a^{i}\left(\frac{b}{a-1}-x_{0}\right) \geq \frac{b}{a-1}
$$

if and only if $a^{i}\left(b /(a-1)-x_{0}\right) \leq b$. But, by (50), $a^{i}(b /(a-$ 1) $\left.-x_{0}\right) \leq b / a^{k-i} \leq b$. Therefore, $x_{i} \geq b /(a-1)>\lambda$ and $x_{i+j}=f_{+}(i, j)$ for all $j$ by Lemma $5(2)$.

Suppose that $\lambda=b /(a-1)-b / a^{k}$. Then

$$
\begin{aligned}
\frac{b}{a-1}-\frac{b}{a^{2}} & \leq \frac{b}{a-1}-\frac{b}{a^{k}}=\lambda<x_{0}<\frac{b}{a-1}-\frac{b}{a^{k+1}}, \\
\frac{b}{a(a-1)} & \leq \frac{b}{a-1}-\frac{b}{a^{k-1}}<x_{1}=a x_{0}-b \\
& <\frac{b}{a-1}-\frac{b}{a^{k}}=\lambda .
\end{aligned}
$$

Thus $i=2$ and $x_{2}=a x_{1}>a(b / a(a-1))=b /(a-1)$.

(b) Follows immediately from Lemma 6(2).

Note that, for any $\lambda$ in $I_{4} \cup I_{5}$, (5) and (6) cover the following cases:

(i) $x_{0}$ is in $I_{3} \cup I_{4} \cup I_{5}$ whenever $a \geq 2$.

(ii) $x_{0}$ is in $\{b / a(a-1)\} \cup I_{4} \cup I_{5}$ when $a<2$.

(7) Let $\lambda$ be in $I_{4} \cup I_{5}$.

(a) Let $a<2$ and let $x_{0} \neq b / a(a-1)$ be in $I_{3}$. Then $b / a \leq$ $x_{0}<b / a(a-1)<\lambda$ and

$$
\begin{aligned}
\frac{b}{a} & <b=a\left(\frac{b}{a}\right) \leq x_{1}=a x_{0}<a\left(\frac{b}{a(a-1)}\right) \\
& =\frac{b}{a-1} .
\end{aligned}
$$

(i) Assume that $\phi \leq a<2$. Then $a(a-1) \geq 1$ so $b / a(a-$ 1) $\leq b$ and therefore, by (70), $x_{1}$ is in $\{b / a(a-1)\} \cup$ $I_{4} \cup I_{5}$. Hence, (5) or (6) applies to $x_{n}(n \geq 1)$.

(ii) Suppose that $a<\phi$ so that $a(a-1)<1$. By (70), $x_{1}$ is in $I_{3} \cup I_{4} \cup I_{5}$. If $x_{1}$ is in $\{b / a(a-1)\} \cup I_{4} \cup I_{5}$, then (5) or (6) applies to $x_{n}(n \geq 1)$.

Assume $x_{1} \neq b / a(a-1)$ is in $I_{3}$. Then, by $(70), b \leq x_{1}<$ $b / a(a-1)<\lambda$ and

$$
\frac{b}{a}<b<a b \leq x_{2}=a x_{1}<\frac{b}{a-1} .
$$

Hence, $x_{2}$ is in $I_{3} \cup I_{4} \cup I_{5}$. Note that if $x_{2} \neq b / a(a-1)$ is in $I_{3}$, then $a b \leq x_{2}<b / a(a-1)<\lambda$ and $a^{2}(a-1)<1$. 
Continuing by induction, for every $j \geq 1$, either $x_{j}$ is in $\{b / a(a-1)\} \cup I_{4} \cup I_{5}$ or $x_{j} \neq b / a(a-1)$ is in $I_{3}$ and $a^{j}(a-1)<1$. Therefore, since $a>1$, if $r$ is the least positive integer such that $a^{r}(a-1) \geq 1$, then there exists an integer $s$ in $[1, r]$ such that $x_{s}=a^{s} x_{0}$ is in $\{b / a(a-1)\} \cup I_{4} \cup I_{5}$.

(b) Let $a>1$ and let $x_{0}$ be in $I_{2}$. Then $b / a^{2}<x_{0}<b / a(a-$ 1) $<\lambda$ and $b / a<x_{1}=a x_{0}<b /(a-1)$. Therefore, $x_{1}$ is in $I_{4} \cup I_{5}$ when $a \geq 2$; and $x_{1} \neq b / a$ is in $I_{3} \cup I_{4} \cup I_{5}$ otherwise.

(c) Let $x_{0}$ be in $I_{1}$ and let $k \geq 2$ be given by (49). Then, for $j=0,1, \ldots, k-2$,

$$
\frac{b}{a^{k+1-j}}<x_{j}=a^{j} x_{0} \leq \frac{b}{a^{k-j}} \leq \frac{b}{a^{2}}<\lambda .
$$

Moreover, $b / a^{2}<x_{k-1}=a^{k-1} x_{0} \leq b / a<\lambda$ and

$$
\frac{b}{a}<x_{k}=a^{k} x_{0} \leq b
$$

(i) If $a \geq 2$, then, by (73), either $x_{k} \geq b /(a-1)$ or $x_{k}$ is in $I_{4} \cup I_{5}$.

(ii) If $a<2$, then $b<b /(a-1)$ and therefore, by (73), $x_{k} \neq b / a$ is in $I_{3} \cup I_{4} \cup I_{5}$.

\section{Periodic Solutions}

The following sets are basic components of any eventually periodic solution of the difference equation (18).

Definition 14. Let $\mathbb{P}_{-1}(a) \equiv\{0\}$ and, for $k \geq 0$, let $\mathbb{P}_{k}(a)$ be the set of all polynomials in $a$ with degree at most $k$ and with all coefficients either 0 or 1 .

A consequence of the next result is that if $x_{m}=x_{m+p}$, then there are $2^{m+p}$ possibilities for $x_{0}$.

Lemma 15. Let $x_{n}$ be defined by (18) with $a>1, b>0$, and $0<\lambda, x_{0}<b /(a-1)$. If $x_{m}=x_{m+p}$, for some integers $m \geq 0$ and $p \geq 1$, then there exist polynomials $\mathbf{p}_{p-1}(a)$ in $\mathbb{P}_{p-1}(a)$ and $\mathbf{q}_{m-1}(a)$ in $\mathbb{P}_{m-1}(a)$ such that

$$
x_{0}=\frac{b\left(\mathbf{p}_{p-1}(a)+\left(a^{p}-1\right) \mathbf{q}_{m-1}(a)\right)}{a^{m}\left(a^{p}-1\right)} .
$$

Proof. Note that $\mathbb{P}_{0}(a)=\{0,1\}$ and, for $k \geq 1$,

$$
\mathbb{P}_{k}(a)=\left\{\sum_{j=0}^{k} \mathbf{p}_{0}^{(k-j)}(a) a^{j}: \mathbf{p}_{0}^{(k-j)}(a) \in \mathbb{P}_{0}(a) \forall j\right\} .
$$

We solve the equation $x_{m}=x_{m+p}$ for general initial value $x_{0}$.

The possible expressions for $x_{m+1}$ may be written as follows:

$$
x_{m+1}=a x_{m}-\mathbf{p}_{0}^{(0)}(a) b, \quad \mathbf{p}_{0}^{(0)}(a) \in \mathbb{P}_{0}(a) .
$$

Similarly,

$$
\begin{aligned}
x_{m+2} & =a x_{m+1}-\mathbf{p}_{0}^{(1)}(a) b \\
& =a^{2} x_{m}-\left(\mathbf{p}_{0}^{(1)}(a)+a \mathbf{p}_{0}^{(0)}(a)\right) b,
\end{aligned}
$$

where $\mathbf{p}_{0}^{(1)}(a) \in \mathbb{P}_{0}(a)$.

Continuing in this manner, we have

$$
x_{m+p}=a^{p} x_{m}-\mathbf{p}_{p-1}(a) b,
$$

where $\mathbf{p}_{p-1}(a)=\sum_{j=0}^{p-1} \mathbf{p}_{0}^{(p-1-j)}(a) a^{j}$ is an arbitrary element of $\mathbb{P}_{p-1}(a)$.

In particular,

$$
x_{m}=a^{m} x_{0}-\mathbf{q}_{m-1}(a) b, \quad \mathbf{q}_{m-1}(a) \in \mathbb{P}_{m-1}(a) .
$$

Therefore, $x_{m}=x_{m+p}$ is equivalent to

$$
x_{m}=\frac{\mathbf{p}_{p-1}(a) b}{a^{p}-1}=a^{m} x_{0}-\mathbf{q}_{m-1}(a) b
$$

and thus the desired form (74) for $x_{0}$ follows.

Note that the initial value of the unbounded solution of Example 8 is not of form (74).

Our main results are converses of Lemma 15. If $x_{0}$ is defined by (74), then

$$
\begin{aligned}
0 & \leq x_{0} \\
& \leq \frac{b\left(\left(a^{p}-1\right) /(a-1)+\left(a^{p}-1\right)\left(\left(a^{m}-1\right) /(a-1)\right)\right)}{a^{m}\left(a^{p}-1\right)} \\
& =\frac{b}{a-1} .
\end{aligned}
$$

Furthermore, we have the following refinement.

Lemma 16. Let $a>1$ and let $b>0$ and suppose that $x_{0}$ satisfies (74) for integers $m \geq 0$ and $p \geq 1$ and polynomials $\mathbf{p}_{p-1}(a)$ in $\mathbb{P}_{p-1}(a)$ and $\mathbf{q}_{m-1}(a)$ in $\mathbb{P}_{m-1}(a)$.

(1) If

$$
\begin{aligned}
\operatorname{deg} \mathbf{q}_{m-1}(a) & <m-1 \quad \text { when } m>1, \\
\mathbf{q}_{m-1}(a) & =0 \quad \text { when } m=1, \\
\operatorname{deg} \mathbf{p}_{p-1}(a) & <p-1 \quad \text { when } m=0, p>1, \\
\mathbf{p}_{p-1}(a) & =0 \quad \text { when } m=0, p=1,
\end{aligned}
$$

then $0 \leq x_{0} \leq b / a(a-1)$.

(2) If

$$
\begin{aligned}
\operatorname{deg} \mathbf{q}_{m-1}(a) & =m-1 \quad \text { when } m>1, \\
\mathbf{q}_{m-1}(a) & =1 \quad \text { when } m=1, \\
\operatorname{deg} \mathbf{p}_{p-1}(a) & =p-1 \quad \text { when } m=0, p>1, \\
\mathbf{p}_{p-1}(a) & =1 \quad \text { when } m=0, p=1,
\end{aligned}
$$

then $b / a \leq x_{0} \leq b /(a-1)$.

Moreover, the converses of (1) and (2) hold whenever $x_{0}$ is not in $I_{3}$. 
Proof. Suppose that $m>1$ and $\operatorname{deg} \mathbf{q}_{m-1}(a)<m-1$. Then $0 \leq$ $\mathbf{q}_{m-1}(a) \leq\left(a^{m-1}-1\right) /(a-1)$ and $0 \leq \mathbf{p}_{p-1}(a) \leq\left(a^{p}-1\right) /(a-1)$ and thus, by (74),

$$
\begin{aligned}
0 & \leq x_{0} \\
& \leq \frac{b\left(\left(a^{p}-1\right) /(a-1)+\left(a^{p}-1\right)\left(\left(a^{m-1}-1\right) /(a-1)\right)\right)}{a^{m}\left(a^{p}-1\right)} \\
& =\frac{b}{a-1} .
\end{aligned}
$$

Similarly, if $m>1$ and $\operatorname{deg} \mathbf{q}_{m-1}(a)=m-1$, then $\mathbf{q}_{m-1}(a) \geq a^{m-1}$ and, by (74) and (81),

$$
\frac{b}{a-1} \geq x_{0} \geq \frac{b\left(\mathbf{p}_{p-1}(a)+\left(a^{p}-1\right) a^{m-1}\right)}{a^{m}\left(a^{p}-1\right)} \geq \frac{b}{a} .
$$

Suppose that $m=1$. Then $\mathbb{P}_{m-1}(a)=\{0,1\}$. If $\mathbf{q}_{m-1}(a)=$ 0 , then, by (74),

$$
0 \leq x_{0}=\frac{b}{a(a-1)} \frac{\mathbf{p}_{p-1}(a)}{\left(\left(a^{p}-1\right) /(a-1)\right)} \leq \frac{b}{a(a-1)} .
$$

And if $\mathbf{q}_{m-1}(a)=1$, then

$$
\frac{b}{a-1} \geq x_{0}=\frac{b\left(\mathbf{p}_{p-1}(a)+\left(a^{p}-1\right)\right)}{a\left(a^{p}-1\right)} \geq \frac{b}{a} .
$$

Assume next that $m=0$ and $p>1$. Then $\mathbb{P}_{m-1}(a)=\{0\}$. If $\operatorname{deg} \mathbf{p}_{p-1}(a)<p-1$, then $\mathbf{p}_{p-1}(a) \leq\left(a^{p-1}-1\right) /(a-1)$ and

$$
0 \leq x_{0} \leq b \frac{\mathbf{p}_{p-1}(a)}{a^{p}-1} \leq \frac{b}{a-1}\left(\frac{a^{p-1}-1}{a^{p}-1}\right)<\frac{b}{a(a-1)}
$$

since $a>1$. And if $\operatorname{deg} \mathbf{p}_{p-1}(a)=p-1$, then $\mathbf{p}_{p-1}(a) \geq a^{p-1}$ and

$$
\frac{b}{a-1} \geq x_{0}=b \frac{\mathbf{p}_{p-1}(a)}{a^{p}-1} \geq \frac{b a^{p-1}}{a^{p}-1}>\frac{b}{a} .
$$

Finally assume that $m=0$ and $p=1$. Then $\mathbb{P}_{m-1}(a)=$ $\{0\}$. If $\mathbf{p}_{p-1}(a)=0$, then $x_{0}=0$. And if $\mathbf{p}_{p-1}(a)=1$, then $x_{0}=b /(a-1)$.

Conversely, suppose that $0 \leq x_{0} \leq b / a(a-1)$ and $m>1$ but $x_{0}$ is not in $I_{3}$. Either $\operatorname{deg} \mathbf{q}_{m-1}(a)<m-1$ or $\operatorname{deg} \mathbf{q}_{m-1}(a)=$ $m-1$. If $\operatorname{deg} \mathbf{q}_{m-1}(a)=m-1$, then $b / a \leq x_{0} \leq b /(a-1)$ by (2). Thus, in this case, if $a<2$, then $x_{0}$ is in $I_{3}$; and if $a \geq 2$, then $a=2$ and $x_{0}$ is again in $I_{3}$. Hence, $\operatorname{deg} \mathbf{q}_{m-1}(a)<m-1$.

The other converses follow similarly.

The converses in Lemma 16 may fail when $x_{0}$ is in $I_{3}$ : in Example 12, we have $a<2, x_{m+p-q-1}=b a^{p-1} /\left(a^{p}-1\right)$ is in $I_{3}, 0 \leq x_{m+p-q-1} \leq b / a(a-1)$, and $x_{m+p-q-1}$ satisfies (74) with $m=0$ and $p>1$ but $\operatorname{deg} \mathbf{p}_{p-1}(a)=p-1$.

If $\lambda$ is in $I_{1} \cup I_{5}$, then, by Theorem 13, $x_{n}$ either has a stationary state or is unbounded. Using Lemma 16, we may extend this result to the other cases of $\lambda$.
Theorem 17. Let $x_{n}$ be a solution of (18) such that $a \geq 2, b>0$, and $x_{0}$ satisfies (74) for some integers $m \geq 0$ and $p \geq 1$ and some polynomials $\mathbf{p}_{p-1}(a)$ in $\mathbb{P}_{p-1}(a)$ and $\mathbf{q}_{m-1}(a)$ in $\mathbb{P}_{m-1}(a)$.

(1) If $\lambda$ is in $I_{2}$, then either $x_{m}=x_{m+p}$ or there exists a positive integer $i \leq m+p$ such that $x_{i+j}=f_{-}(i, j)$ for all $j \geq 0$.

(2) Let $\lambda$ be in $I_{3}$. If $\lambda \neq b / a$ or $\mathbf{p}_{p-1}(a) \neq 0$, then $x_{m}=$ $x_{m+p}$. On the other hand, if $\lambda=b / a$ and $\mathbf{p}_{p-1}(a)=0$, then either $x_{0}=0$ or there exists a positive integer $i \leq m$ such that $x_{i+j}=f_{+}(i, j)$ for all $j \geq 0$, where $x_{i}=b$.

(3) If $\lambda$ is in $I_{4}$, then either $x_{m}=x_{m+p}$ or there exists a positive integer $i \leq m+p$ such that $x_{i+j}=f_{+}(i, j)$ for all $j \geq 0$.

Proof. (2) Let $\lambda$ be in $I_{3}$ and suppose that either $\lambda \neq b / a$ or $\mathbf{p}_{p-1}(a) \neq 0$. We first verify that, for $m \geq 1$,

$$
x_{m}=\frac{b \mathbf{p}_{p-1}(a)}{a^{p}-1} .
$$

Suppose that $\mathbf{q}_{m-1}(a)=0$. Then

$$
\begin{aligned}
x_{0} & =\frac{b}{a-1} \frac{\mathbf{p}_{p-1}(a)}{a^{m}\left(1+a+\cdots+a^{p-1}\right)} \leq \frac{b}{a-1} \frac{1}{a^{m}} \\
& \leq \frac{b}{a-1} \frac{1}{a} \leq \lambda
\end{aligned}
$$

since $a \geq 2$ and $\lambda$ is in $I_{3}$. Thus $x_{1}=a x_{0}$ and similarly $x_{j}=$ $a x_{j-1} \leq \lambda$ for $j=1, \ldots, m-1$. Hence, $x_{m}=a^{m} x_{0}$ satisfies $(90)$.

Next assume $\mathbf{q}_{m-1}(a) \neq 0$ and $m=1$. Since $a \geq 2$ and $\lambda$ is in $I_{3}$, it follows that

$$
x_{0}=\frac{b\left(\mathbf{p}_{p-1}(a)+\left(a^{p}-1\right)\right)}{a\left(a^{p}-1\right)} \geq \frac{b}{a} \geq \lambda .
$$

Additionally, since $\lambda<b / a$ or $\mathbf{p}_{p-1}(a)>0$, at least one of the inequalities is strict and $x_{0}>\lambda$. Thus $x_{1}=a x_{0}-b$ satisfies (90).

Hence, assume that $\mathbf{q}_{m-1}(a) \neq 0$ and $m>1$. There exists a nonempty subset $\Lambda_{m-1}$ of $\{0,1, \ldots, m-1\}$ such that

$$
x_{0}=b\left(\frac{\mathbf{p}_{p-1}(a)}{a^{m}\left(a^{p}-1\right)}+\frac{\mathbf{q}_{m-1}(a)}{a^{m}}\right),
$$

where $\mathbf{q}_{m-1}(a)=\sum_{k \in \Lambda_{m-1}} a^{k}$. Let $i \equiv \min \Lambda_{m-1}$ and $j \equiv$ $\max \Lambda_{m-1}=\operatorname{deg} \mathbf{q}_{m-1}(a)$. We will show that

$$
x_{m-i}=\frac{b \mathbf{p}_{p-1}(a)}{a^{i}\left(a^{p}-1\right)}
$$

and therefore (90) will hold as in the initial case $\mathbf{q}_{m-1}(a)=0$.

We begin by showing that, for $m>1$,

$$
x_{0}>\lambda \text { iff } j=m-1 .
$$

Let $x_{0}>\lambda$. If $a=2$, then $b / a(a-1)=\lambda=b / a<x_{0}$ and therefore $x_{0}$ is not in $I_{3}$. On the other hand, if $a>2$, then $b / a(a-1)<b / a$ and, by Lemma $16, x_{0}$ is again not in $I_{3}$. 
Since $b / a(a-1) \leq \lambda \leq b / a, x_{0}>\lambda$, and $x_{0}$ is not in $I_{3}$, it follows that $b / a<x_{0} \leq b /(a-1)$ and, by Lemma $16, j=m-1$.

Conversely, let $j=m-1$. Then $\mathbf{q}_{m-1}(a) \geq a^{m-1}$ and

$$
x_{0} \geq b\left(\frac{\mathbf{p}_{p-1}(a)}{a^{m}\left(a^{p}-1\right)}+\frac{a^{m-1}}{a^{m}}\right) \geq \frac{b}{a} \geq \lambda \text {. }
$$

Since $\lambda<b / a$ or $\mathbf{p}_{p-1}(a)>0$, at least one of the latter inequalities is strict and thus $x_{0}>\lambda$. Hence, (95) holds.

Next consider $x_{1}$, where $\mathbf{q}_{m-1}(a) \neq 0$ and $x_{0}$ is given by (93). By (95),

$$
x_{1}=b\left(\frac{\mathbf{p}_{p-1}(a)}{a^{m-1}\left(a^{p}-1\right)}+\frac{\mathbf{q}_{m-2}(a)}{a^{m-1}}\right),
$$

where $\mathbf{q}_{m-2}(a)=\sum_{k \in \Lambda_{m-2}} a^{k}$ such that

$$
\Lambda_{m-2} \equiv \begin{cases}\Lambda_{m-1}, & \text { if } j<m-1 \\ \Lambda_{m-1} \backslash\{j\}, & \text { if } j=m-1,\end{cases}
$$

which is a subset of $\Lambda_{m-1} \cap\{0, \ldots, m-2\}$. In addition, since $i \leq j \leq m-1$, it follows that

$$
\mathbf{q}_{m-2}(a)=0 \text { iff } 1=m-i,
$$

in which case (94) holds for $x_{1}$.

Suppose that $1<m-i$. Then $\mathbf{q}_{m-2}(a) \neq 0, i$ is in $\Lambda_{m-2}$, and $x_{1}$ is of form (93) with $m$ replaced by $m-1$. By (95),

$$
x_{1}>\lambda \text { iff } \max \Lambda_{m-2}=m-2 .
$$

Moreover, as above, (94) holds for $x_{2}$ if $2=m-i$.

By induction, for $r=0,1, \ldots, m-i-1$, we may assume

$$
x_{r}=b\left(\frac{\mathbf{p}_{p-1}(a)}{a^{m-r}\left(a^{p}-1\right)}+\frac{\mathbf{q}_{m-r-1}(a)}{a^{m-r}}\right) \text {, }
$$

where $\mathbf{q}_{m-r-1}(a)=\sum_{k \in \Lambda_{m-r-1}} a^{k} \neq 0$ such that

$$
\begin{aligned}
& \Lambda_{m-r-1} \\
& \equiv \begin{cases}\Lambda_{m-r}, & \text { if } \max \Lambda_{m-r}<m-r \\
\Lambda_{m-r} \backslash\left\{\max \Lambda_{m-r}\right\}, & \text { if } \max \Lambda_{m-r}=m-r,\end{cases}
\end{aligned}
$$

which is a subset of $\Lambda_{m-r} \cap\{0, \ldots, m-r-1\}$, and, by (95),

$$
x_{r}>\lambda \text { iff } \max \Lambda_{m-r-1}=m-r-1
$$

Moreover, after $r \equiv m-i$ steps, we conclude that $\mathbf{q}_{m-r-1}(a)=0$ and $x_{m-i}$ satisfies (94). Thus (90) follows as in the case $\mathbf{q}_{m-1}(a)=0$.

Finally, we verify that $x_{m}=x_{m+p}$, where $x_{m}$ is given by (90). If $p=1$, then $x_{m}$ is 0 or $b /(a-1)$; so $x_{m}=x_{m+p}$. Thus assume $p>1$. In this case, we prove that

$$
x_{m} \leq \lambda \text { iff } \operatorname{deg} \mathbf{p}_{p-1}(a)<p-1 .
$$

Let $x_{m} \leq \lambda$. Suppose first that $x_{m}$ is in $I_{3}$. Since $a \geq 2$, we have $b / a(a-1) \leq b / a$; and, by Lemma $16, b / a(a-1)=x_{m}=$ $\lambda=b / a$ and $a=2$. Hence, by (90), $\mathbf{p}_{p-1}(a)=\left(a^{p}-1\right) / a$, which is impossible since $a=2$ and $\mathbf{p}_{p-1}(a)$ is a positive integer but $a^{p}-1$ and $a$ are relatively prime.

Thus assume $x_{m}$ is not in $I_{3}$. Then $x_{m}$ is in $I_{1} \cup I_{2}$ since $\lambda$ is in $I_{3}$ and $x_{m} \leq \lambda$. Hence, by Lemma $16, \operatorname{deg} \mathbf{p}_{p-1}(a)<p-1$ since $p>1$.

Conversely, let $\operatorname{deg} \mathbf{p}_{p-1}(a)<p-1$ (and $p>1$ ). Then $0 \leq x_{m} \leq b / a(a-1) \leq \lambda$ by Lemma 16 and therefore $x_{m} \leq \lambda$. Thus (104) follows.

As in (93), by (90),

$$
x_{m}=\frac{b \sum_{k \in \Lambda_{p-1}} a^{k}}{a^{p}-1}
$$

for some nonempty subset $\Lambda_{p-1}$ of $\{0, \ldots, p-1\}$. By (104),

$$
x_{m+1}=\frac{b \sum_{k \in \Lambda_{p-1}} T_{p-1}\left(a^{k}\right)}{a^{p}-1},
$$

where $T_{p-1}$ is the mapping on the set $\left\{a^{0}, a^{1}, \ldots, a^{p-1}\right\}$ defined by

$$
T_{p-1}\left(a^{k}\right) \equiv \begin{cases}a^{k+1}, & \text { if } k<p-1 \\ a^{0}, & \text { if } k=p-1 .\end{cases}
$$

Similarly, for $r=1,2, \ldots$,

$$
x_{m+r}=\frac{b \sum_{k \in \Lambda_{p-1}} T_{p-1}^{r}\left(a^{k}\right)}{a^{p}-1}
$$

and since $T_{p-1}^{p}\left(a^{k}\right)=a^{k}$, for all $k$, it follows that $x_{m}=x_{m+p}$.

We now turn to the second part of (2). Assume that $a \geq 2$, $\lambda=b / a$, and $x_{0}=b \mathbf{q}_{m-1}(a) / a^{m}>0$ and thus $m \geq 1$. We prove the following:

$$
\text { If } x_{0}=\frac{b \mathbf{Q}_{j}(a)}{a^{k}} \neq 0 \quad \text { where } \mathbf{Q}_{j}(a) \text { is in } \mathbb{P}_{j}(a)
$$

such that $0 \leq j=\operatorname{deg} \mathbf{Q}_{j}(a)<k \leq m$,

then either $x_{0}=\frac{b}{a}=\lambda$

or $x_{1}=\frac{b \mathbf{Q}_{j^{\prime}}(a)}{a^{k-1}} \neq 0$

where $\mathbf{Q}_{j^{\prime}}(a)$ is in $\mathbb{P}_{j^{\prime}}(a)$ and $0 \leq j^{\prime}=\operatorname{deg} \mathbf{Q}_{j^{\prime}}(a)<k-1 \leq m$.

Suppose that $x_{0}=b \mathbf{Q}_{j}(a) / a^{k} \neq 0$, where $\mathbf{Q}_{j}(a)$ satisfies the above hypotheses. Consider two cases for $j$.

(i) Assume $j \leq k-2$. Then $x_{0}=b \mathbf{Q}_{j}(a) / a^{k}<\lambda=b / a$ : $\mathbf{Q}_{j}(a) \leq 1+a+\cdots+a^{j}=\left(a^{j+1}-1\right) /(a-1)<a^{k-1}$ since

$$
\begin{aligned}
a^{k-1}+a^{j+1}-1 & \leq a^{k-1}+a^{k-1}-1=2 a^{k-1}-1 \\
& \leq a a^{k-1}-1<a^{k} .
\end{aligned}
$$

Hence, (109) holds since $x_{1}=a x_{0}=b \mathbf{Q}_{j}(a) / a^{k-1} \neq 0$, where $0 \leq j=\operatorname{deg} \mathbf{Q}_{j}(a) \leq k-2<k-1 \leq m$ so let $j^{\prime}=j$. 
(ii) Suppose that $j=k-1$. If $\mathbf{Q}_{j}(a)=a^{j}$, then $x_{0}=b / a=$ $\lambda$ so assume that $\mathbf{Q}_{j}(a)>a^{j}$ since $j=\operatorname{deg} \mathbf{Q}_{j}(a)$. In this case, (109) also holds since $x_{0}=b \mathbf{Q}_{j}(a) / a^{k}>\lambda=b / a$ and hence $x_{1}=a x_{0}-b=b \mathbf{Q}_{j^{\prime}}(a) / a^{k-1} \neq 0$, where $\mathbf{Q}_{j^{\prime}}(a) \equiv \mathbf{Q}_{j}(a)-a^{j}$ and $0 \leq j^{\prime} \equiv \operatorname{deg} \mathbf{Q}_{j^{\prime}}(a) \leq k-2<k-1 \leq m$.

Thus, starting with $x_{0}=b \mathbf{q}_{m-1}(a) / a^{m}=b \mathbf{Q}_{j}(a) / a^{k} \neq 0$, $\mathbf{Q}_{j}(a) \in \mathbb{P}_{j}(a), 0 \leq j=\operatorname{deg} \mathbf{Q}_{j}(a)<k=m$, and, applying (109) to $x_{1}, x_{2}, \ldots$, we conclude that $x_{i-1}=b / a=\lambda$ for some $i$ in $[1, m]$. Therefore,

$$
x_{i}=a x_{i-1}=b \geq \frac{b}{a-1}>\frac{b}{a}=\lambda
$$

and $x_{i+j}=f_{+}(i, j)$ for all $j \geq 0$ by Lemma $5(2)$.

(1) Let $\lambda$ be in $I_{2}$. The condition " $x_{i+j}=f_{-}(i, j)$ for all $j \geq$ 0 " is equivalent to " $x_{i} \leq 0$." Thus we further assume that $x_{i}>$ 0 for $i=1, \ldots, m+p$ and show that $x_{m}=x_{m+p}$ following the outline of the proof of (2).

We first verify that $x_{m}$ satisfies (90) for $m \geq 1$. Suppose that $\mathbf{q}_{m-1}(a)=0$. Then, since $a \geq 2$ and $m \geq 1$,

$$
\begin{aligned}
x_{0} & =\frac{b}{a-1} \frac{\mathbf{p}_{p-1}(a)}{a^{m}\left(\left(a^{p}-1\right) /(a-1)\right)} \leq \frac{b}{a-1} \frac{1}{a^{m}} \\
& \leq \frac{b}{a-1} \frac{1}{a} \leq \frac{b}{a} .
\end{aligned}
$$

If $x_{0}>\lambda$, then $x_{1} \leq 0$ by Lemma $6(4)$. Therefore, $x_{0} \leq \lambda$ and

$$
x_{1}=a x_{0}=\frac{b \mathbf{p}_{p-1}(a)}{a^{m-1}\left(a^{p}-1\right)} .
$$

Similarly, $x_{j} \leq \lambda$ for $j=1, \ldots, m-1$ and hence $x_{m}=a^{m} x_{0}$ satisfies (90).

Suppose next that $\mathbf{q}_{m-1}(a) \neq 0$ and $m=1$. Since $\lambda$ is in $I_{2}$,

$$
x_{0}=\frac{b\left(\mathbf{p}_{p-1}(a)+\left(a^{p}-1\right)\right)}{a\left(a^{p}-1\right)} \geq \frac{b}{a}>\lambda
$$

and $x_{1}=a x_{0}-b$ satisfies (90).

Thus assume that $\mathbf{q}_{m-1}(a) \neq 0$ and $m>1$. Then $x_{0}$ is given by (93), where $\mathbf{q}_{m-1}(a)=\sum_{k \in \Lambda_{m-1}} a^{k}$ for some nonempty subset $\Lambda_{m-1}$ of $\{0,1, \ldots, m-1\}$. We will show that (94) holds, where $i \equiv \min \Lambda_{m-1}$, and therefore (90) will hold as in the case $\mathbf{q}_{m-1}(a)=0$.

Let $j \equiv \max \Lambda_{m-1}=\operatorname{deg} \mathbf{q}_{m-1}(a)$. We begin by showing (95) for $m>1$.

Let $x_{0}>\lambda$. If $x_{0}$ is in $(\lambda, b / a(a-1)) \cup I_{3}$, then $x_{1} \leq 0$ by Lemma 6(4). Since $x_{1}>0$ by assumption and $x_{0}>\lambda$, it follows that $x_{0}$ is in $I_{4} \cup I_{5}$; and therefore $j=m-1$ by Lemma 16 since $m>1$.

Conversely, let $j=m-1$. By Lemma 16,

$$
\lambda<\frac{b}{a(a-1)} \leq \frac{b}{a} \leq x_{0} \leq \frac{b}{a-1}
$$

and $x_{0}>\lambda$. Thus (95) holds.

Equation (94) and consequently (90) follow from (95) as in the proof of (2).
Finally, we verify that $x_{m}=x_{m+p}$, where $x_{m}$ is given by (90). We may assume $p>1$ since otherwise $x_{m}$ is 0 or $b /(a-1)$, and therefore $x_{m}=x_{m+p}$. We start by proving (104): since $\lambda$ is in $I_{2}$ and $x_{m+1}>0$ by assumption, it follows by Lemma 6(4) that $x_{m}$ is not in $(\lambda, b / a(a-1)) \cup I_{3}$.

Let $x_{m} \leq \lambda$. Then $x_{m}$ is in $I_{1} \cup I_{2} \operatorname{sodeg} \mathbf{p}_{p-1}(a)<p-1$ by Lemma 16 since $x_{m}$ is not in $I_{3}$ and $p>1$.

Conversely, assume that $\operatorname{deg} \mathbf{p}_{p-1}(a)<p-1$. By Lemma $16,0 \leq x_{m} \leq b / a(a-1)$, but $x_{m}$ is not in $(\lambda, b / a(a-1)]$. Therefore $x_{m} \leq \lambda$ and (104) holds.

By (90) and (104), the desired result $x_{m}=x_{m+p}$ follows as in the proof of (2).

(3) Let $\lambda$ be in $I_{4}$. The condition " $x_{i+j}=f_{+}(i, j)$ for all $j \geq 0$ " is equivalent to " $x_{i} \geq b /(a-1)$." Thus we assume that $x_{i}<b /(a-1)$ for $i=1, \ldots, m+p$ and show that $x_{m}=x_{m+p}$ following the outline of the proof of (2).

We first verify that $x_{m}$ satisfies (90) for $m \geq 1$. Suppose that $\mathbf{q}_{m-1}(a)=0$. Then, since $a \geq 2, m \geq 1$, and $\lambda$ is in $I_{4}$,

$$
\begin{aligned}
x_{0} & =\frac{b}{a-1} \frac{\mathbf{p}_{p-1}(a)}{a^{m}\left(\left(a^{p}-1\right) /(a-1)\right)} \leq \frac{b}{a-1} \frac{1}{a^{m}} \\
& \leq \frac{b}{a-1} \frac{1}{a} \leq \frac{b}{a}<\lambda .
\end{aligned}
$$

Thus $x_{1}=a x_{0}$ and similarly $x_{j} \leq \lambda$ for $j=1, \ldots, m-1$. Hence, $x_{m}=a^{m} x_{0}$ satisfies (90).

Next, we assume $\mathbf{q}_{m-1}(a) \neq 0$ and $m=1$. Then

$$
x_{0}=\frac{b\left(\mathbf{p}_{p-1}(a)+\left(a^{p}-1\right)\right)}{a\left(a^{p}-1\right)} \geq \frac{b}{a} \geq \frac{b}{a(a-1)} .
$$

If $x_{0} \leq \lambda$, then $x_{1} \geq b /(a-1)$ by Lemma 6(2), contrary to our hypothesis. Thus $x_{0}>\lambda$ and $x_{1}=a x_{0}-b$ satisfies (90).

Therefore assume that $\mathbf{q}_{m-1}(a) \neq 0$ and $m>1$. Then $x_{0}$ is given by (93), where $\mathbf{q}_{m-1}(a)=\sum_{k \in \Lambda_{m-1}} a^{k}$ for some nonempty subset $\Lambda_{m-1}$ of $\{0,1, \ldots, m-1\}$. We will show that (94) holds, where $i \equiv \min \Lambda_{m-1}$, and hence (90) will follow as in the case $\mathbf{q}_{m-1}(a)=0$.

Let $j \equiv \max \Lambda_{m-1}=\operatorname{deg} \mathbf{q}_{m-1}(a)$. We begin by showing (95) for $m>1$ : if $x_{0}$ is in $I_{3} \cup(b / a, \lambda)$, then $x_{1} \geq b /(a-1)$ by Lemma 6(2). Thus $x_{0}$ is not in $I_{3} \cup(b / a, \lambda)$ by our hypothesis.

Let $x_{0}>\lambda$. Then $x_{0}$ is in $I_{4} \cup I_{5}, m>1$, and hence $j=m-1$ by Lemma 16 since $x_{0}$ is not in $I_{3}$.

Conversely, let $j=m-1$ (and $m>1$ ). By Lemma 16, $b / a(a-1) \leq b / a \leq x_{0} \leq b /(a-1)$. Since $x_{0}$ is not in $(b / a, \lambda)$, we have that $x_{0}>\lambda$; and (95) holds.

Equation (94) and consequently (90) follow from (95) as in the proof of (2).

Finally, we verify that $x_{m}=x_{m+p}$, where $x_{m}$ is given by (90). We may assume $p>1$ since otherwise $x_{m}$ is 0 or $b /(a-$ 1 ), and therefore $x_{m}=x_{m+p}$. We start by proving (104).

Let $x_{m} \leq \lambda$. Then $x_{m}$ is not in $I_{3} \cup(b / a, \lambda)$ by Lemma 6(2) since $x_{m+1}<b /(a-1)$. Thus $x_{m}$ is in $I_{1} \cup I_{2}$ and therefore $\operatorname{deg} \mathbf{p}_{p-1}(a)<p-1$ by Lemma 16 since $x_{m}$ is not in $I_{3}$ and $p>1$.

Conversely, if $\operatorname{deg} \mathbf{p}_{p-1}(a)<p-1$ (and $p>1$ ), then $0 \leq$ $x_{m} \leq b / a(a-1)<\lambda$ by Lemma 16, and (104) follows. 
The following two examples illustrate symmetry between type $C$ solutions about the point $b / 2(a-1)$ that will be generalized in the next section. In particular, type $\mathrm{B}$ solutions will be shown to be reflections of type A solutions.

Example 18. Let $a \geq 2$ and let $b>0$ and let $\lambda$ be in $I_{3}$. For positive integer $k$, consider the weighted average

$$
x_{0} \equiv \frac{b / a^{2}+a^{k}(b / a(a-1))}{1+a^{k}}
$$

in $I_{2}$ (that converges to $b / a(a-1)$ as $k$ tends to infinity). Then

$$
x_{0}=\frac{b\left(a^{k}\left(\left(a^{k}-1\right) /(a-1)\right)+\left(a^{2 k}-1\right)\right)}{a^{2}\left(a^{2 k}-1\right)}
$$

so $x_{2}=x_{2+2 k}$ by Theorem $17(2)$.

Example 19. For integer $k \geq 1$, redefine $x_{0}$ in Example 18 to be the weighted average

$$
x_{0} \equiv \frac{\left(b /(a-1)-b / a^{2}\right)+a^{k}(b / a)}{1+a^{k}}
$$

in $I_{4}$ (that converges to $b / a$ as $k \rightarrow \infty$ ). Then $x_{2}=x_{2+2 k}$ as above since

$$
x_{0}=\frac{b\left(\left(a^{k}-1\right) /(a-1)+\left(a^{2 k}-1\right) a\right)}{a^{2}\left(a^{2 k}-1\right)}
$$

in form (74). Note that, in this case, $b /(a-1)-x_{0}$ is the initial value in Example 18.

Theorem 17 may be applied to cryptography.

Example 20. Arbitrary vectors $\mathbf{v}=\left\langle v_{0}, \ldots, v_{p-1}\right\rangle$ of zeros and ones may be encoded by selecting any real numbers $a \geq 2$, $b>0$, and $\lambda$ in $I_{3}$ and defining

$$
x_{0} \equiv \frac{b \sum v_{i} a^{i}}{a^{p}-1}
$$

in form (74). By Theorem 17(2), the "cipher-set" $\left\{a, b, \lambda, x_{0}\right.$ (computed value) $\}$ may then be decoded for $\mathbf{v}$ as follows: if $x_{0}=0$, then $\mathbf{v}$ is a zero vector of indeterminate length. If $x_{0}=b /(a-1)$, then $\mathbf{v}$ is a vector of all ones of indeterminate length.

Assume $x_{0} \neq 0, b /(a-1)$. Compute enough terms of the periodic sequence $x_{n}$ to determine its period $p$. Then $p$ is the length of $\mathbf{v}$ and the proof of Theorem 17(2) shows that

$$
v_{i}=1 \text { iff } x_{p-1-i}>\lambda \quad(i=0, \ldots, p-1) .
$$

The following eventually periodic solution $x_{n}$ with $x_{0}$ of form (74) is of type A.

Example 21. Let $a \geq 2$ and let $b>0$ and, for integers $m \geq 1$ and $p>2$, let $x_{0}$ be the weighted average

$$
x_{0} \equiv \frac{b a^{p-2} /\left(a^{p}-1\right)+\left(a^{m-1}-1\right)(b / a(a-1))}{a^{m-1}}
$$

in $I_{2}$. Then $x_{0}$ is of form (74) with $\mathbf{p}_{p-1}(a)=a^{p-1}$ and $\mathbf{q}_{m-1}(a)=\left(a^{m-1}-1\right) /(a-1)$. Choose $\lambda$ in $I_{2}$ such that $x_{0} \leq \lambda$. Then $x_{m}=b a^{p-1} /\left(a^{p}-1\right)$ :

$$
x_{1}=a x_{0}=\frac{b a^{p-1}}{a^{m-1}\left(a^{p}-1\right)}+\frac{b}{a^{m-1}}\left(\frac{a^{m-1}-1}{a-1}\right),
$$

so the claim is true for $m=1$.

Assume $m>1$. We prove by induction that

$$
x_{i}=\frac{b a^{p-1}}{a^{m-i}\left(a^{p}-1\right)}+\frac{b}{a^{m-i}}\left(\frac{a^{m-i}-1}{a-1}\right)>\frac{b}{a}>\lambda
$$

for $i=1, \ldots, m-1$. By the form of $x_{1}$ above,

$$
x_{1}>\frac{b}{a^{m-1}}\left(\frac{a^{m-1}-1}{a-1}\right) \geq \frac{b}{a}>\lambda
$$

since $a^{m-1} \geq a$; so (126) holds for $i=1$.

Suppose that (126) is true for some $i<m-1$. Then

$$
\begin{aligned}
x_{i+1} & =a x_{i}-b \\
& =\frac{b a^{p-1}}{a^{m-(i+1)}\left(a^{p}-1\right)}+\frac{b}{a^{m-(i+1)}}\left(\frac{a^{m-(i+1)}-1}{a-1}\right) \\
& \geq \frac{b}{a^{m-(i+1)}}\left(\frac{a^{m-(i+1)}-1}{a-1}\right) \geq \frac{b}{a}>\lambda
\end{aligned}
$$

as in the case $i=1$ since $m>i+1$. Thus (126) holds for $i=1, \ldots, m-1$.

In particular, $x_{m}=a x_{m-1}-b=b a^{p-1} /\left(a^{p}-1\right)>b / a>\lambda$.

Finally $x_{m+p}=x_{m}: x_{m+1}=a x_{m}-b=b /\left(a^{p}-1\right) \leq b / a^{2}<$ $\lambda$ since $p>2$, and, by inducton, $x_{m+i}=b a^{i-1} /\left(a^{p}-1\right) \leq$ $b / a^{2}<\lambda$ for $i=1, \ldots, p-2$. Hence, $x_{m+(p-1)}=a x_{m+(p-2)}=$ $b a^{p-2} /\left(a^{p}-1\right)<x_{0} \leq \lambda$ by the form of $x_{0}$ and the choice of $\lambda$. Therefore, $x_{m+p}=b a^{p-1} /\left(a^{p}-1\right)=x_{m}$.

Unfortunately, not all type A solutions $x_{n}$ with $x_{0}$ of form (74) are bounded.

Example 22. Let $a \geq 2$ and let $b>0$ and

$$
\begin{aligned}
\frac{b}{a^{2}} & <x_{0} \equiv \frac{b\left(a^{2}+1\right)}{a\left(a^{3}-1\right)}<\lambda \equiv \frac{b a}{(a-1)\left(a^{2}+1\right)} \\
& <\frac{b}{a(a-1)} .
\end{aligned}
$$

Then $x_{0}$ is of form (74) with $m=1, p=3, \mathbf{p}_{p-1}(a)=a^{2}+1$, and $\mathbf{q}_{m-1}(a)=0$. Moreover,

$$
\begin{aligned}
& x_{1}=a x_{0}=\frac{b\left(a^{2}+1\right)}{a^{3}-1}>\frac{b}{a}>\lambda, \\
& x_{2}=a x_{1}-b=\frac{b(a+1)}{a^{3}-1}>\lambda, \\
& x_{3}=a x_{2}-b=\frac{b\left(\left(a^{3}-1\right) /(a-1)-a^{3}\right)}{a^{3}-1}<0
\end{aligned}
$$

since $a \geq 2$. Therefore, $x_{3+j}=f_{-}(3, j)$ for all $j \geq 0$. 
Suppose that $x_{0}$ is defined by (74). By Theorems 13 and 17 , if $a \geq 2$ and $x_{n}$ is bounded, then $x_{n}$ is eventually periodic. In fact, if $a=2$ and $\lambda$ is in $I_{3}$, then $x_{m}=x_{m+p}$ by treating the cases $\mathbf{p}_{p-1}(a) \neq 0$ and $\mathbf{p}_{p-1}(a)=0$ separately. However, if $a<2$ and $\lambda$ is in $I_{3}$, then $x_{n}$ is bounded (Lemma 6) but may not be eventually periodic.

Example 23. Assume that $1<a=r / s<\phi$, where $r$ and $s$ are odd and even integers, respectively; $b>0$ and $x_{0} \equiv$ $b a /\left(a^{2}-1\right)$. Then $x_{0}$ is of form (74) with $m=0, p=2$, and $\mathbf{p}_{p-1}(a)=a$; and $x_{0}$ is in $I_{3}$ since $a(a-1)<1$. Choose $\lambda$ in $I_{3}$ such that $x_{0} \leq \lambda$. Then $\lambda \neq b / a$ and $\mathbf{p}_{p-1}(a) \neq 0$, but $x_{n}$ is not eventually periodic: with $r_{0} \equiv r$ and $r_{1} \equiv r^{2}$, we have as in Example 9, for $n \geq 0$,

$$
x_{n}=\frac{b}{a-1} \frac{r_{n}}{s^{n}(r+s)},
$$

where $r_{n}$ is odd. If $x_{n}$ is eventually periodic, then $x_{u}=x_{u+v}$ for some $u \geq 0$ and $v \geq 1$; and therefore $r_{u+v}=s^{v} r_{u}$ is both even and odd.

\section{Symmetric Solutions}

Theorems 13 and 17 indicate symmetry about the midpoint of $(0, b /(a-1))$ between pairs of solutions of (18). Moreover, if $x_{0}$ satisfies (74), then so does $b /(a-1)-x_{0}$ : if

$$
x_{0}=\frac{b\left(\mathbf{p}_{p-1}(a)+\left(a^{p}-1\right) \mathbf{q}_{m-1}(a)\right)}{a^{m}\left(a^{p}-1\right)}
$$

then

$$
\frac{b}{a-1}-x_{0}=\frac{b\left(\left(\left(a^{p}-1\right) /(a-1)-\mathbf{p}_{p-1}(a)\right)+\left(a^{p}-1\right)\left(\left(a^{m}-1\right) /(a-1)-\mathbf{q}_{m-1}(a)\right)\right)}{a^{m}\left(a^{p}-1\right)} .
$$

Therefore, in view of Theorem 17, a natural question is, if $x_{n}$ and $y_{n}$ are given by (18), where $y_{0} \equiv b /(a-1)-x_{0}$, does it follow that $y_{n}=b /(a-1)-x_{n}$ for all $n$ ? We show affirmative answers in general for at least two choices of $\lambda$, the first of which requires that $\lambda$ is not a term of $x_{n}$.

Theorem 24. Let $x_{n}$ satisfy difference equation (18) with respect to $a>1, b>0,0<\lambda, x_{0}<b /(a-1)$, and $F_{\lambda} \equiv F$; and let $y_{n}$ be defined by (18) in terms of $a \equiv a, b \equiv b$, $\lambda_{y} \equiv b /(a-1)-\lambda, y_{0} \equiv b /(a-1)-x_{0}$, and the corresponding $F$ denoted by $F_{\lambda_{y}}$. Then

$$
y_{1}= \begin{cases}\frac{b}{a-1}-x_{1}, & \text { if } x_{0} \neq \lambda \\ a\left(\frac{b}{a-1}\right)-x_{1}, & \text { if } x_{0}=\lambda .\end{cases}
$$

Moreover, if $x_{0}=\lambda$ and $\lambda \geq b / a(a-1)$, then $x_{1+j}=f_{+}(1, j)$ for all $j \geq 0$.

Proof. Suppose first that $x_{0}>\lambda$. Then $x_{1}=a x_{0}-b=b /(a-$ $1)-a\left(b /(a-1)-x_{0}\right)$ and $y_{0}=b /(a-1)-x_{0}<b /(a-1)-\lambda=\lambda_{y}$ so that $y_{1}=a y_{0}=b /(a-1)-x_{1}$.

Next, assume that $x_{0} \leq \lambda$. Then $x_{1}=a x_{0}$. If $x_{0}<\lambda$, then $y_{0}=b /(a-1)-x_{0}>b /(a-1)-\lambda=\lambda_{y}$ and therefore $y_{1}=a y_{0}-b=b /(a-1)-a x_{0}=b /(a-1)-x_{1}$. And if $x_{0}=\lambda$, then $y_{0}=b /(a-1)-\lambda=\lambda_{y}$ and $y_{1}=a y_{0}=a(b /(a-1))-x_{1}$.

Finally, if $x_{0}=\lambda \geq b / a(a-1)$, then $x_{1+j}=f_{+}(1, j)$ by Lemma 6(2).

For the solutions $x_{n}$ in the following examples (with the conditions imposed), $y_{n}=b /(a-1)-x_{n}$ for all $n$ by Theorem 24 since $x_{k} \neq \lambda$ for all $k$ : Examples 8 (if $\lambda \neq$ $b / 2(a-1)), 12,21$ (if $x_{0}<\lambda$ ), and 22 .
Let $x_{n}$ and $y_{n}$ be given as in Theorem 24 and suppose further that $x_{k} \neq \lambda$ for all $k$. By Remark 2, $x_{n}=x(n)$, where $x=x(t)$ satisfies

$$
x^{\prime}(t)=A x(t)+B x([t])+C F_{\lambda}(x([t]))
$$

for constants $A, B$, and $C$ such that $b /(a-1)=-C /(A+$ $B)$. Therefore, $y_{n}=y(n)$, where $y(t) \equiv b /(a-1)-x(t)$ is the symmetric solution about the line $x=b / 2(a-1)$ of the differential equation

$$
y^{\prime}(t)=A y(t)+B y([t])+C F_{\lambda_{y}}(y([t]))
$$

with $y(0)=b /(a-1)-x_{0}$; and $F_{\lambda_{y}}(y([t]))=1-F_{\lambda}(x([t]))$ since $x([t]) \neq \lambda$, and hence $x([t]) \leq \lambda$ if and only if $y([t])>$ $\lambda$.

For type $\mathrm{C}$ solutions, where $\lambda \neq b / a$, there may be another symmetric solution obtained by reflecting $x_{0}$ about $b / 2(a-1)$.

Theorem 25. Let $x_{n}$ satisfy (18) with respect to $a>2, b>0$, and $0<x_{0}<b /(a-1)$ such that either

(1) $b / a(a-1) \leq \lambda<b / 2(a-1)$ and $x_{n}$ is bounded below or

(2) $b / 2(a-1) \leq \lambda<b / a$ and $x_{n}$ is bounded above.

If $z_{n}$ satisfies (18) in terms of $a \equiv a, b \equiv b, \lambda \equiv \lambda$, and $z_{0} \equiv b /(a-1)-x_{0}$, then $z_{n}=b /(a-1)-x_{n}$ for all $n$.

Proof. Suppose that (1) holds. If $x_{0} \leq \lambda$, then $x_{1}=a x_{0}$ and $z_{0}=b /(a-1)-x_{0} \geq b /(a-1)-\lambda>b /(a-1)-b / 2(a-1)=$ $b / 2(a-1)>\lambda$. Thus, $z_{1}=a z_{0}-b=b /(a-1)-a x_{0}=$ $b /(a-1)-x_{1}$ in this case. 
Next, assume that $x_{0}>\lambda$. Then $x_{1}=a x_{0}-b=b /(a-1)-$ $a\left(b /(a-1)-x_{0}\right)=b /(a-1)-a z_{0}$. We claim that $z_{0} \leq \lambda$. By way of contradiction, assume that $z_{0}>\lambda \geq b / a(a-1)$. Then $x_{0}=b /(a-1)-z_{0}<b /(a-1)-\lambda \leq b /(a-1)-b / a(a-1)=b / a$. Hence, $x_{1}=a x_{0}-b<a(b / a)-b=0$ and $x_{n}$ is not bounded below by Lemma 5(1), contrary to (1). Therefore $z_{0} \leq \lambda$ and $z_{1}=a z_{0}=b /(a-1)-x_{1}$.

It follows in either case that $z_{1}=b /(a-1)-x_{1}$; and, by induction, $z_{n}=b /(a-1)-x_{n}$ for all $n$.

Suppose now that (2) holds. If $x_{0}>\lambda$, then $x_{1}=a x_{0}-b$ and $z_{0}=b /(a-1)-x_{0}<b /(a-1)-\lambda \leq b /(a-1)-b / 2(a-$ $1)=b / 2(a-1) \leq \lambda$. Thus $z_{1}=a z_{0}=a b /(a-1)-a x_{0}=$ $a b /(a-1)-\left(x_{1}+b\right)=b /(a-1)-x_{1}$ in this case.

Finally assume that $x_{0} \leq \lambda$. We claim that $z_{0}>\lambda$. Suppose otherwise that $z_{0} \leq \lambda$. Then $x_{0}=b /(a-1)-z_{0} \geq b /(a-$ 1) $-\lambda>b /(a-1)-b / a=b / a(a-1)$ and $x_{1}=a x_{0}>$ $a(b / a(a-1))=b /(a-1)$; so $x_{n}$ is not bounded above by Lemma 5(2), contradicting (2). Therefore $z_{0}>\lambda$ and $z_{1}=$ $a z_{0}-b=b /(a-1)-a x_{0}=b /(a-1)-x_{1}$.

Hence, $z_{1}=b /(a-1)-x_{1}$ in both cases; and, by induction, $z_{n}=b /(a-1)-x_{n}$ for all $n$.

Note that Theorem 25 may fail when $\lambda=b / a$ : let $a>2$ and let $b>0$ and let $x_{0} \equiv b / a=\lambda$. Then $x_{1}=a x_{0}=b$ and $z_{0}=b /(a-1)-x_{0}=b / a(a-1)<\lambda$. Thus $z_{1}=a z_{0}=$ $b /(a-1) \neq b /(a-1)-x_{1}=b /(a-1)-b$.

The solution $x_{n}$ in Example 11 satisfies $y_{n}=b /(a-1)-x_{n}$ for all $n$ whenever $\lambda \neq b a^{p-2} /\left(a^{p}-1\right)$ by Theorem 24 since $\lambda$ is thus not a term of $x_{n}$. Furthermore, in this example, if $a>2$ and $\lambda \neq b / a$ is in $I_{3}$, then $z_{n}=b /(a-1)-x_{n}$ for all $n$ by Theorem 25 since $x_{n}$ is eventually periodic and therefore bounded.

Let $x_{n}$ and $z_{n}$ be given as in Theorem 25. By Remark 2, $x_{n}=x(n)$, where $x=x(t)$ satisfies (2) for constants $A, B$, and $C$ such that $b /(a-1)=-C /(A+B)$. Therefore, $z_{n}=z(n)$, where $z(t) \equiv b /(a-1)-x(t)$ is the symmetric solution about $x=b / 2(a-1)$ of the same differential equation

$$
z^{\prime}(t)=A z(t)+B z([t])+C F(z([t]))
$$

but with $z(0)=b /(a-1)-x_{0}$, since, by the proof of Theorem 25, $F(z([t]))=1-F(x([t]))$ (i.e., $x([t]) \leq \lambda$ if and only if $z([t])>\lambda)$.

If $x_{0}$ is given by (74), then $b /(a-1)-x_{0}$ is given by (133) and Theorem 17 may be applied to Theorems 24 and 25 .

Corollary 26. Let $x_{n}$ satisfy (18), where $a \geq 2, b>0, \lambda$ is in $I_{3}$, and $x_{0}$ is given by (74) for some integers $m \geq 0$ and $p \geq 1$ and some polynomials $\mathbf{p}_{p-1}(a)$ in $\mathbb{P}_{p-1}(a)$ and $\mathbf{q}_{m-1}(a)$ in $\mathbb{P}_{m-1}(a)$.

(1) If $\lambda \neq b / a(a-1)$ or $\mathbf{p}_{p-1}(a) \neq\left(a^{p}-1\right) /(a-1)$ or $a=2$, then $y_{m}=y_{m+p}$. Moreover, if $x_{n}$ is bounded with no stationary states, then $y_{n}=b /(a-1)-x_{n}$ for all $n$. In particular, if $\mathbf{p}_{p-1}(a) \neq 0,\left(a^{p}-1\right) /(a-1)$, then $y_{n}=b /(a-1)-x_{n}$ for all $n$.

(2) If $\lambda \neq b / a$ or $\mathbf{p}_{p-1}(a) \neq\left(a^{p}-1\right) /(a-1)$, then $z_{m}=$ $z_{m+p}$. Moreover, if $a>2, \lambda \neq b / a$, and $x_{n}$ is bounded, then $z_{n}=b /(a-1)-x_{n}$ for all $n$.
Proof. Suppose that $a \geq 2, b>0, \lambda$ is in $I_{3}$, and $x_{0}$ is given by (74). The first lines of (1) and (2) follow directly from (133) and Theorem 17(2).

(1) Assume $x_{n}$ is bounded with no stationary states. Then $x_{0} \neq 0, b /(a-1)$ and $0<x_{0}<b /(a-1)$ by (81). By Theorem 13, $x_{k}$ is not in $I_{3}$ so $x_{k} \neq \lambda$ for all $k$. Thus $y_{n}=b /(a-1)-x_{n}$ by Theorem 24

In particular, suppose that $\mathbf{p}_{p-1}(a) \neq 0,\left(a^{p}-1\right) /(a-$ $1)$. Then $x_{n}$ is eventually periodic, and hence bounded, by Theorem 17(2); and, by the proof of this theorem, the only possible stationary states are when

(i) $\mathbf{q}_{m-1}(a)=0$ and $x_{0}=b \mathbf{p}_{p-1}(a) / a^{m}\left(a^{p}-1\right)=0$ (s $b / a(a-1))$ or

(ii) $x_{m}=b \mathbf{p}_{p-1}(a) /\left(a^{p}-1\right)$ is 0 or $b /(a-1)$,

that is, when $\mathbf{p}_{p-1}(a)$ is 0 or $\left(a^{p}-1\right) /(a-1)$, which are ruled out. Thus, by the previous case, $y_{n}=b /(a-1)-x_{n}$ for all $n$.

(2) Next, assume that $a>2, \lambda \neq b / a$, and $x_{n}$ is bounded. By (81), $0 \leq x_{0} \leq b /(a-1)$. If $x_{0}=0$, then $x_{n}=0$ for all $n$; and hence $z_{0}=b /(a-1)$ and thus $z_{n}=b /(a-1)=b /(a-1)-x_{n}$ for all $n$. Similarly, if $x_{0}=b /(a-1)$, then $z_{n}=b /(a-1)-x_{n}$ for all $n$.

Therefore, we may assume $0<x_{0}<b /(a-1)$ and the desired result is now immediate from Theorem 25 .

Example 18 (revisited). In this example, $a \geq 2, b>0, \lambda$ is in $I_{3}$, and $x_{0}$ is of form (74) with $m=2, p=2 k \geq 2, \mathbf{p}_{p-1}(a)=$ $a^{k}\left(\left(a^{k}-1\right) /(a-1)\right)$, and $\mathbf{q}_{m-1}(a)=1$. Moreover, $x_{2}=x_{2+2 k}$. By Corollary 26, $y_{n}=b /(a-1)-x_{n}$ for all $n$ and $z_{2}=z_{2+2 k}$. Furthermore, if $a>2$ and $\lambda \neq b / a$, then $z_{n}=b /(a-1)-x_{n}$ for all $n$.

Example 27. Let $a \geq 2$ and let $b>0$ and let $\lambda$ be in $I_{3}$. For positive integer $k$, consider the weighted average

$$
x_{0} \equiv \frac{b / a+a^{k}\left(b /(a-1)-b / a^{2}\right)}{1+a^{k}}
$$

in $I_{4}$ (that converges to $b /(a-1)-b / a^{2}$ as $k$ tends to infinity). Then $x_{0}$ is of form (74) with $m=1, p=2 k \geq 2, \mathbf{p}_{p-1}(a)=$ $a^{k-1}\left(\left(a^{k}-1\right) /(a-1)\right)$, and $\mathbf{q}_{m-1}(a)=1$ so $x_{1}=x_{1+2 k}$ by Theorem 17(2). Therefore, as above,

(i) $y_{n}=b /(a-1)-x_{n}$ for all $n$;

(ii) $z_{1}=z_{1+2 k}$;

(iii) if $a>2$ and $\lambda \neq b / a$, then $z_{n}=b /(a-1)-x_{n}$ for all $n$.

Moreover,

$$
\begin{aligned}
& \frac{b}{a-1}-x_{0}=\frac{b / a(a-1)+a^{k}\left(b / a^{2}\right)}{1+a^{k}} \\
& =\frac{b\left(\left(\left(a^{k}-1\right) /(a-1)\right)\left(a^{k}-a^{k-1}+1\right)+\left(a^{2 k}-1\right) 0\right)}{a\left(a^{2 k}-1\right)} .
\end{aligned}
$$




\section{Conflict of Interests}

The author declares that there is no conflict of interests regarding the publication of this paper.

\section{Acknowledgment}

The author would like to thank the referee(s) for several helpful suggestions.

\section{References}

[1] S. M. Shah and J. Wiener, "Advanced differential equations with piecewise constant argument deviations," International Journal of Mathematics and Mathematical Sciences, vol. 6, no. 4, pp. 671703, 1983.

[2] K. L. Cooke and J. Wiener, "Retarded differential equations with piecewise constant delays," Journal of Mathematical Analysis and Applications, vol. 99, no. 1, pp. 265-297, 1984.

[3] A. R. Aftabizadeh, J. Wiener, and J.-M. Xu, "Oscillatory and periodic solutions of delay differential equations with piecewise constant argument," Proceedings of the American Mathematical Society, vol. 99, no. 4, pp. 673-679, 1987.

[4] A. Cabada and J. B. Ferreiro, "First order differential equations with piecewise constant arguments and nonlinear boundary value conditions," Journal of Mathematical Analysis and Applications, vol. 380, no. 1, pp. 124-136, 2011.

[5] J. Hong, R. Obaya, and A. Sanz, "Almost periodic type solutions of some differential equations with piecewise constant argument," Nonlinear Analysis: Theory, Methods \& Applications, vol. 45, no. 6, pp. 661-688, 2001.

[6] G. Wang, "Existence theorem of periodic solutions for a delay nonlinear differential equation with piecewise constant arguments," Journal of Mathematical Analysis and Applications, vol. 298, no. 1, pp. 298-307, 2004.

[7] R. Yuan, "The existence of almost periodic solutions of retarded differential equations with piecewise constant argument," Nonlinear Analysis: Theory, Methods \& Applications, vol. 48, no. 7, pp. 1013-1032, 2002.

[8] Z. Zhou, "Periodic orbits on discrete dynamical systems," Computers \& Mathematics with Applications, vol. 45, no. 6-9, pp. 1155-1161, 2003.

[9] Y. Chen, "All solutions of a class of difference equations are truncated periodic," Applied Mathematics Letters, vol. 15, no. 8, pp. 975-979, 2002.

[10] C. Hou and S. S. Cheng, "Eventually periodic solutions for difference equations with periodic coefficients and nonlinear control functions," Discrete Dynamics in Nature and Society, vol. 2008, Article ID 179589, 21 pages, 2008.

[11] T. Yi and Z. Zhou, "Periodic solutions of difference equations," Journal of Mathematical Analysis and Applications, vol. 286, no. 1, pp. 220-229, 2003.

[12] H. Zhu, L. Huang, and X. Liao, "Convergence and periodicity of solutions for a class of delay difference equations," Computers and Mathematics with Applications, vol. 48, no. 10-11, pp. 14771484, 2004.

[13] S. Al-Ashhab and J. Guyker, "Piecewise defined recursive sequences with application in matrix theory," Journal of Mathematical and Computational Science, vol. 2, no. 4, pp. 793-809, 2012. 


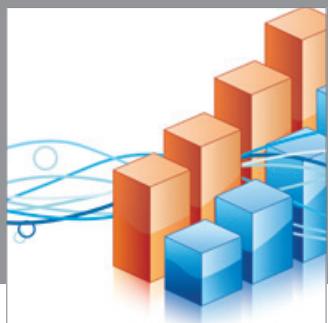

Advances in

Operations Research

mansans

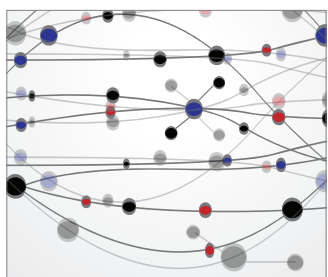

The Scientific World Journal
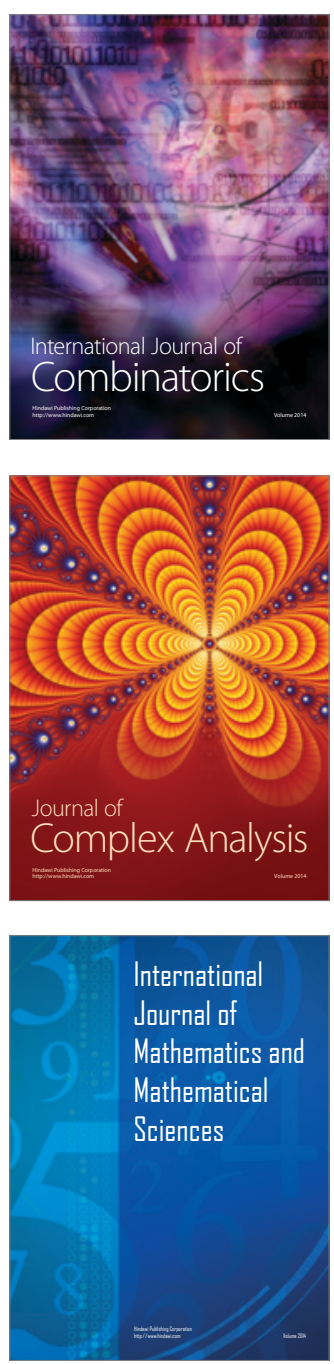
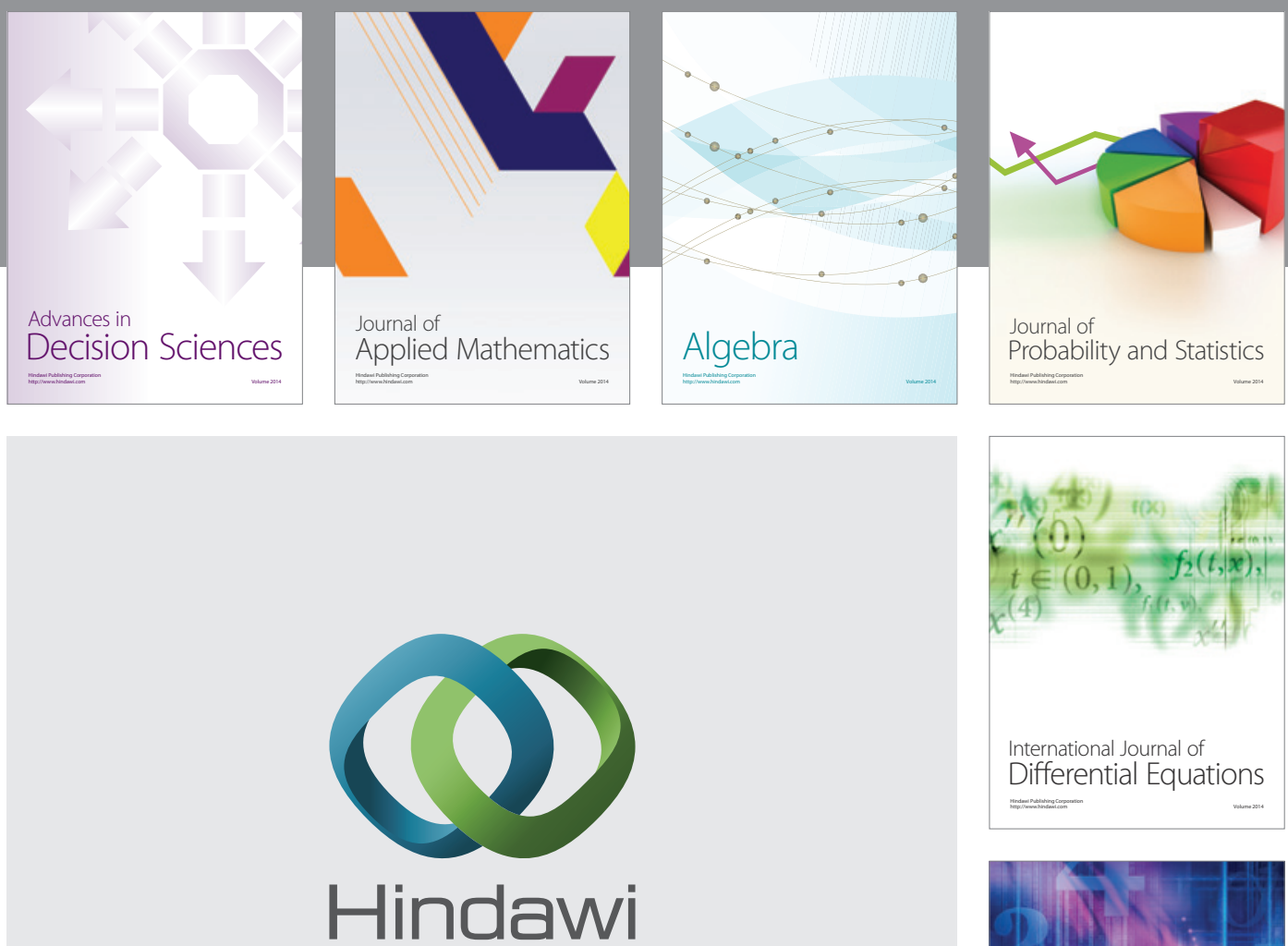

Submit your manuscripts at http://www.hindawi.com
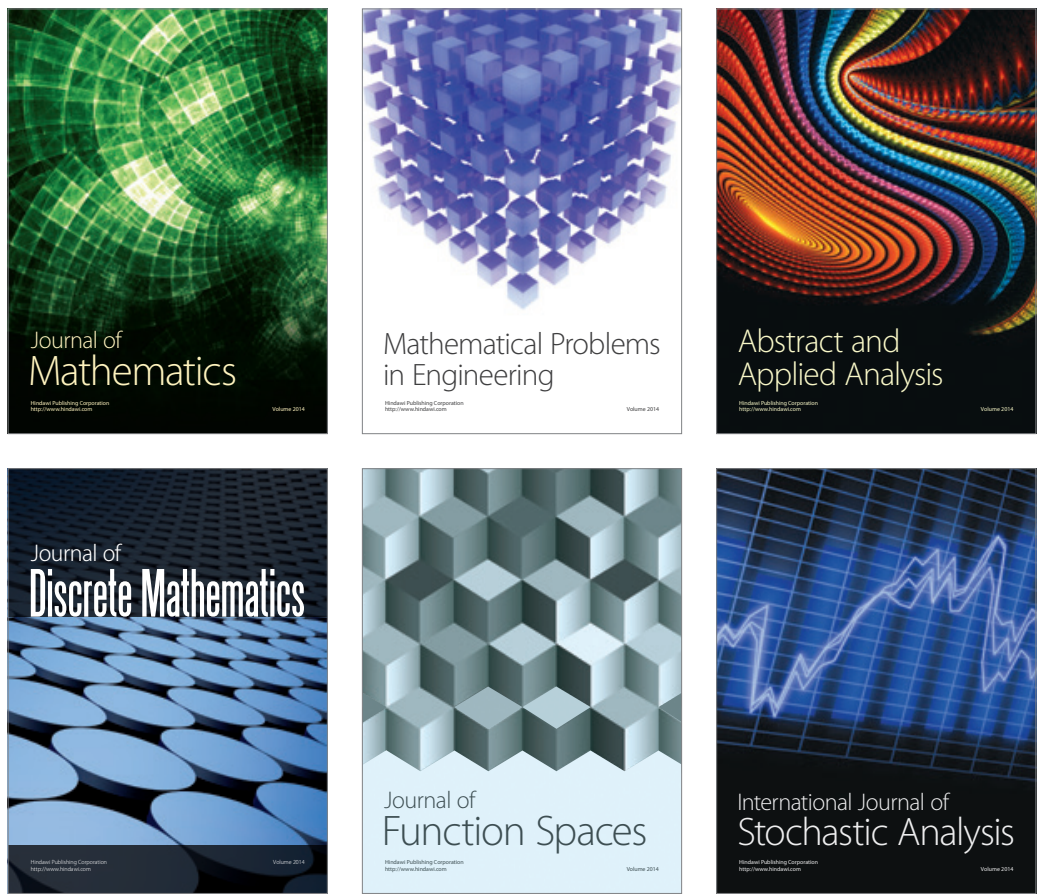

Journal of

Function Spaces

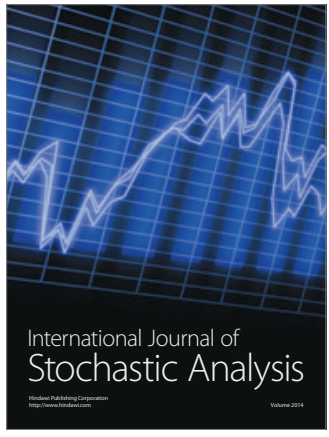

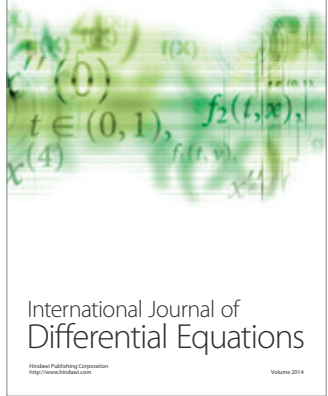
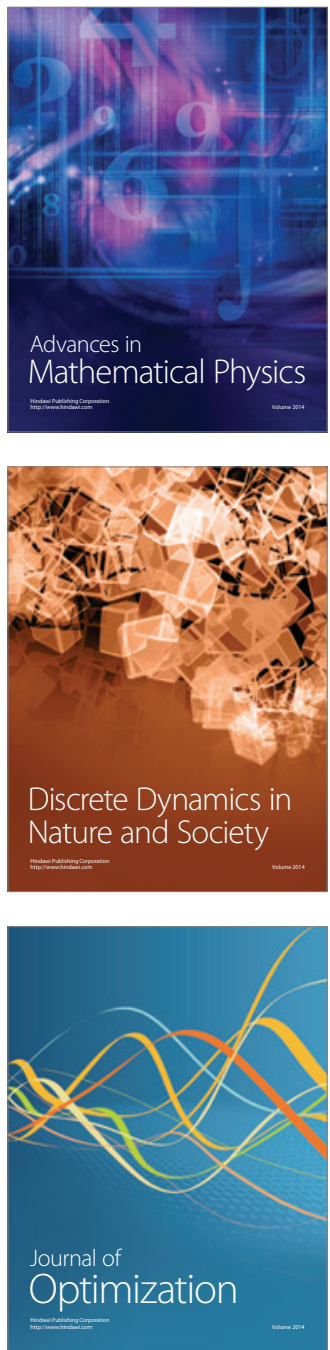